\title{
DEVELOPMENT OF QUADCOPTER FOR SEARCH AND RESCUE IN NATURAL DISASTERS
}

A project report submitted in the partial fulfillment of the requirement for the award of the degree of

\section{BACHELOR OF TECHNOLOGY}

in

ELECTRONICS \& INSTRUMENTATION ENGINEERING

by

E.GOPI KRISHNA (09016T0616)

S.NAGARAJU (09016T0655)

A.VAMSI KRISHNA (09016T0619)

B.MAHESH BABU (09016T0645)

Under the guidance of

Sri K.SRINIVAS

Assistant Professor, Dept. of E\&I Engg.

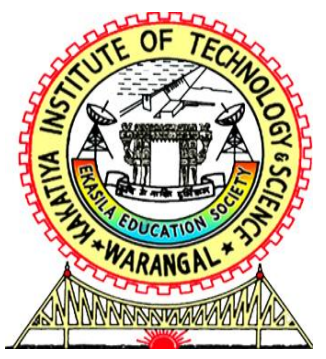

DEPARTMENT OF ELECTRONICS \& INSTRUMENTATION ENGINEERING

KAKATIYA INSTITUTE OF TECHNOLOGY \& SCIENCE (Affiliated to Kakatiya University)

WARANGAL-506015

March 2013 
dedicated to our department (E\&I Engg.) 


\section{Acknowledgement}

No creation in this world is solo effort. Neither is this project. Well, to say, this is our project would be totally untrue. Albeit this was our dream, there are some people in this world, some of them so wonderful, that made this dream become true. From the person who inspired us to do the project to the person who guided us to complete the project, everyone has a role.

We wish to thank Dr. K.Ashoka Reddy, Principal, Kakatiya Institute of Technology and Science, for providing all the required facilities and encouraging us to do the project by providing fund from college.

Our sincere thanks to Smt M.Sreelatha, Professor \& Head of the Department, E\&I Engineering for providing all the facilities to carry out the project work and her constant encouragement throughout this work.

We express our deep sense of gratitude to our project guide Sri K.Srinivas, Assistant Professor, Dept of E\&I Engg. under whose guidance and supervision this work has been accomplished. His keen interest, constant support has been of great help to us throughout the course of this project work.

We also owe a great deal of thanks to Sri. B. Shashikanth, Asst. Professor, Dept of E\&I Engg for his valuable advice and guidance as project co-ordinator

We thank all the faculty members of Dept. of E\&I Engg for their kind cooperation and valuable help in the completion of the project

God, who continues to look after us despite our flaws.

Our friends who constantly encouraged us to do the project.

And last but not least, our parents for their constant support and encouragement. 


\begin{abstract}
In many circumstances it is desirable to have a versatile, inexpensive robot available to complete a task. This is most often advantageous when a circumstance presents a danger to human life or when a human is not possible due to size or maneuverability issues and also when disasters and crises arise visual information needs to be rapidly gathered and assessed in order to assist rescue workers and emergency personnel. Often such situations are life-threatening and people cannot safely obtain such information. Our goal will be to create an aerial platform that will be the core for mission specific uses. These uses range from surveillance, mapping, search and rescue, security and more. Military applications are also possible and advantageous due to the low cost of the platform and the life saving possibilities of robotic warfare.
\end{abstract}

In the above context we developed an aerial vehicle capable of flight to maneuver down a hallway and send video wirelessly.

To achieve our specified goal and to overcome the above said danger to human life we designed an aerial vehicle (Quad copter). In short terms a Quad Copter is what the name says a copter-like device with four rotors (quad).

Here to achieve the control, the microcontroller is programmed with a main algorithm (Multiwii) that will control the vehicle using inputs from various sensors. Accordingly the control will be given to four motors which control the flight orientation (pitch, roll and yaw) through Electronic speed controllers (ESC).Here to provide the visual aid of the surroundings of our vehicle we have mounted a camera on it and it will be transmitting the video signal wirelessly to the base station where from our vehicle will be controlled. Here, sensors used by us will automatically change the motor speeds for proper orientation and the movement of our vehicle is controlled at base station using RF communication. 


\section{CONTENTS}

$\begin{array}{lr}\text { 1. Introduction } & 12\end{array}$

$\begin{array}{ll}1.1 \text { Introduction } & 12\end{array}$

$\begin{array}{ll}1.2 \text { Problem Statement } & 14\end{array}$

$\begin{array}{ll}1.3 \text { Project scopes/Constraints } & 14\end{array}$

2. Unmanned Aerial Vehicle (UAV Background and Project motivation

$\begin{array}{ll}\text { 2.1 UAV Background } & 15\end{array}$

$\begin{array}{ll}2.2 \text { Project motivation } & 17\end{array}$

$\begin{array}{lr}\text { 3. } & \text { Methodology } \\ \end{array}$

$\begin{array}{ll}3.1 \text { Introduction } & 19\end{array}$

$\begin{array}{ll}3.2 \text { Flowchart } & 19\end{array}$

3.3 Quadcopter Design Constraints 20

3.4 Quadcopter Movement Mechanism 22

3.5 Quadcopter mathematical modeling 25

4. Technical Specifications 29

4.1 Brushless DC motors 29

$\begin{array}{lll}4.2 & \text { Propellers } & 31\end{array}$

4.3 Electronic Speed controllers 32

$\begin{array}{lll}4.4 & \text { Battery } & 33\end{array}$ 
4.5 Battery Charger

4.6 Voltage Detector

4.7 Transmitter \& Receiver 36

4.8 CCD camera \& Tx-Rx 38

4.9 Flight Control Board 39

4.10 Calculations basing on Selected Components 47

5. Software Used 48

$\begin{array}{lll}5.1 & \text { Arduino } & 48\end{array}$

5.2 MULTIWII CONFIG 2_1 51

6. Electrical Connections

6.1 Introduction $\quad 55$

6.2 Schematic Diagram 56

6.3 Building Quadcopter 56

7. Programming and Configuration 63

$\begin{array}{lll}7.1 & \text { Introduction } & 63\end{array}$

$\begin{array}{lll}7.2 & \text { Programming } & 63\end{array}$

$\begin{array}{lll}7.3 \text { Configuration of Quadcopter } & 67\end{array}$

$\begin{array}{ll}\text { 8. FPV } & 71\end{array}$

$\begin{array}{lll}8.1 & \text { Introduction } & 71\end{array}$

$\begin{array}{lll}8.2 & \text { Equipment } & 72\end{array}$ 
8.3 Radio Frequencies

8.4 Ready to Fly

9. Achievements

9.1 Achievements

9.2 Pictures of flight

9.3 Videos of Flight

75

10. Further Developments and Advancements

10.1 Further Developments

10.2 Advancements

Appendix

80

References

81 


\section{List of figures}

$\begin{array}{lll}2.1 & \text { Global Hawk } & 15\end{array}$

$\begin{array}{lll}2.2 & \text { Micro Air vehicles } & 16\end{array}$

3.1 Flow chart of Quadcopter design 20

$\begin{array}{lll}3.2 & \text { Quadcopter X configuration } & 21\end{array}$

$\begin{array}{lll}3.3 & \text { Quadcopter + configuration } & 21\end{array}$

$\begin{array}{lll}3.4 & \text { Pitch direction of Quadcopter } & 22\end{array}$

3.5 Roll direction of Quadcopter 22

3.6 Yaw direction of Quadcopter 23

3.7 Take-off motion 23

$\begin{array}{lll}3.8 & \text { Landing motion } & 23\end{array}$

$\begin{array}{lll}3.9 & \text { Forward motion } & 24\end{array}$

$\begin{array}{ll}3.10 \text { Backward motion } & 24\end{array}$

3.11 Right motion 24

3.12 Left motion 24

$\begin{array}{ll}3.13 \text { Roll right } 25 & 25\end{array}$

$\begin{array}{ll}3.14 \text { Roll left } & 25\end{array}$

3.15 Schematic of Quadcopter 26

3.16 Angle Movement of Quadcopter 27

4.1 Brushless DC motor 29

4.2 Brushless and brushed motor internal 30

$\begin{array}{lll}4.3 & \text { Propellers } & 31\end{array}$

4.4 ESC 32

4.5 Battery 33 
4.6 Battery charger $\quad 35$

$\begin{array}{lll}4.7 & \text { Voltage detector } & 35\end{array}$

$\begin{array}{lll}4.8 & \text { Tx and Rx } & 36\end{array}$

$\begin{array}{lll}4.9 & \text { How to control? } & 37\end{array}$

4.10 Camera and Tx-Rx 38

4.11 Crius Multiwii SE QuadX Flight Control Board 39

$\begin{array}{ll}4.12 \text { Pinout of ATMEGA328P } & 40\end{array}$

4.13 Block diagram of ATMEGA328P 40

4.14 Digital Gyroscope 41

4.15 Digital accelerometer 43

4.16 Accelerometer example 44

4.17 Accelerometer force vector 44

4.18 Magneto resistive sensor 45

4.19 FTDI basic programmer 46

$\begin{array}{ll}4.20 \text { Schematic of FTDI } & 47\end{array}$

$\begin{array}{lll}4.21 \text { Calculations } & 47\end{array}$

5.1 Arduino logo 48

$\begin{array}{lll}5.2 & \text { Arduino environment } & 49\end{array}$

5.3 Selecting an Arduino Uno/microcontroller 50

5.4 Multiwii configuration screen $\quad 51$

6.1 Schematic diagram 56

6.2 Quadcopter frame 57

6.3 Motors mounted onto the frame 58

6.4 Proper tilted Propellers connected to proper motors 58

6.5 ESC to motor wiring $\quad 59$

6.6 ESC to motor $\quad 59$ 
6.7 Wiring to connect battery to four ESCs 59

6.8 Wiring for calibration $\quad 60$

6.9 Receiver and flight control board connection 61

6.10 Motors and flight control board connection $\quad 62$

$\begin{array}{lll}7.1 & \text { Arduino environment } & 66\end{array}$

$\begin{array}{ll}7.2 \text { Com port selection } & 67\end{array}$

$\begin{array}{lll}7.3 & \text { Upload click } & 67\end{array}$

$\begin{array}{ll}7.4 \text { Multiwiiconfig screen } & 68\end{array}$

$\begin{array}{ll}9.1-9.6 & \text { Snapshots of our Flight } \quad 73-74\end{array}$

9.7-9.10 Some snapshots of our video from camera $\quad$ 74-75

$\begin{array}{lll}10.1 & \text { To increase payload } & 77\end{array}$

$\begin{array}{lll}10.28 \text { formation } & 78\end{array}$

$\begin{array}{lll}10.3 & \text { Swarm of quadcopters } & 79\end{array}$ 


\section{CHAPTER 1 INTRODUCTION}

\subsection{Introduction}

Research and development of unmanned aerial vehicle (UAV) and micro aerial vehicle (MAV) are getting high encouragement nowadays, since the application of UAV and MAV can apply to variety of area such as rescue mission, military, film making, agriculture and others.

Aerial robotics is an emerging field which is very exciting. The first robots were for use in industrial manufacturing, and mostly consisted of stationary variants on robotic arms. The next generation of robots was land-based and years of robotics research went into increasing their agility and purposeful movement on the 2D plane. Now it is becoming possible to make robots with the freedom to roam about all three dimensions. A popular platform for small unmanned aerial vehicles (UAVs) is the quad copter.

Multi-rotor aerial vehicles have become increasingly popular robotic platforms because of their mechanical simplicity, dynamic capabilities, and suitability for both indoors and out-door environments. In particular, there have been many recent advances in the design, control and planning for quad rotors, rotorcrafts with four rotors.

The last decade has seen rapid progress in micro aerial robots, autonomous aerial vehicles that are smaller than 1 meter in scale and $1 \mathrm{~kg}$ or less in mass. Winged aircrafts can range from fixed-wing vehicles to flapping-wing vehicles, the latter mostly inspired by insect flight. Rotorcrafts, including helicopters, coaxial rotor crafts , ducted fans, quad rotors and hex rotors, have proved to be more mature with quad rotors being the most commonly used aerial platform in robotics research labs. 
Of course micro aerial robots have a fundamental payload limitation that is difficult to overcome in many practical applications. However larger payloads can be manipulated and transported by multiple UAVs either using grippers or cables.

Applications such as surveillance or search and rescue that require coverage of large areas or imagery from multiple sensors can be addressed by coordinating multiple UAVs, each with different sensors. The most important and obvious benefit of scaling down in size is the ability of the quad rotor to operate in tightly constrained environments in tight formations. While the payload capacity of the quad rotor falls dramatically, it is possible to deploy multiple quadrotors that cooperate to overcome this limitation. Again, the small size benefits us because smaller vehicles can operate in closer proximity than large vehicles. Another interesting benefit of scaling down is agility.

The military and search and rescue use of unmanned aerial vehicles (UAVs) has grown because of their ability to operate in dangerous locations while keeping their human operators at a safe distance. The larger UAVs also provide a reliable long duration, cost effective, platform for reconnaissance as well as weapons. They have grown to become an indispensable tool for the military.

UAVs for military use were reduced to practice in the mid-1990s when the Global Hawk and the Predator were developed. These were very large fixed wing aircraft with wingspans in the 50-100 foot range. Payloads for these large UAVs included radar, laser designators, cameras, and missile systems. The introduction of these aircraft removed the pilots from harm's way plus added the ability to remain in the target area for many hours at a time. However, these UAVs are large and very expensive and they beg the question of whether smaller UAVs could also play a role in military applications. Likewise, on the other extreme, there is considerable work in micro UAVs some of which are bio-inspired designs. There are designs modeled after insects and birds, but just as the large military UAVs are too expensive, we felt that these micro-UAVs were too small to be practical and required technology that was not readily available. It was therefore a vehicle in the one foot to one meter class size that caught our team's interest and is the basis for our project. Specifically, our team is 
very interested in whether these smaller UAVs can be used not only for military applications but also for search and rescue operations. Although most of the large military UAVs are fixed wing aircraft, we felt that a small UAV should have greater maneuverability and versatility since it was likely to be useful for a broader range of applications than the larger or smaller versions. We were also motivated by the DARPA UAVforge challenge which required a vertical takeoff UAV design. We selected the Quadcopter design because of its maneuverability, stability, and large payload capacity. The UAV that we are building is a prototype unit that could be used for rescue operations use but is not rugged or robust enough for military use. Although we met the goal of producing a small UAV that could perform useful missions in both military and rescue operations, time and funding constraints forced us to design a UAV to meet our functional requirements but not to meet harsh environmental conditions such as those encountered during military missions. However, our UAV design certainly could be re-implemented with newer and more robust technology which would allow it to be used for military functions.

\subsection{Problem Statement}

To develop an aerial vehicle capable of flight to maneuver and send video wirelessly.

\subsection{Project scopes/constraints}

The scope includes weather, distance and space:

(a) Quadcopter only can operate in sunny day or dry condition.

(b) Quadcopter operates distance not more than $300 \mathrm{~m}$ in eye sight from the Wireless receiver.

(c) Quadcopter is control by Arduino base microcontroller.

(d) Quadcopter is operated by brushless motor control by electronic speed controller. 


\section{CHAPTER 2}

\section{UNMANNED AERIAL VEHICLES (UAV) BACKGROUND AND PROJECT MOTIVATION}

\subsection{UAV Background}

UAVs for military use were reduced to practice in the mid-1990s with the High-Altitude Endurance Unmanned Aerial Vehicle Advanced Concept Technology Demonstrator (HAE UAV ACTD) program managed by the Defense Advanced Research Projects Agency (DARPA) and Defense Airborne Reconnaissance Office (DARO).This ACTD laid the groundwork for the development of the Global Hawk shown in Figure (2.1).

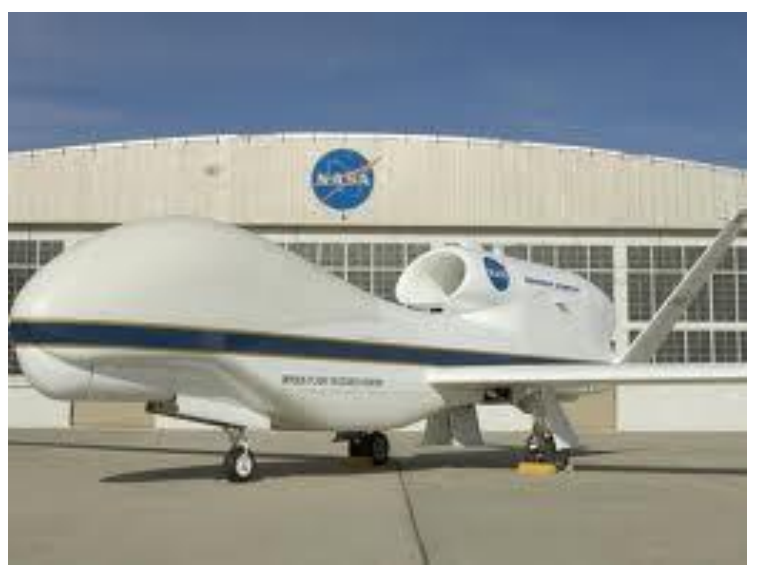

Figure 2.1 Global Hawk

The Global Hawk flies at altitudes up to 65,000 feet for up to 35 hours at speeds approaching 340 knots while costing approximately 200 million dollars. The wingspan is 116 feet and it can fly 12,000 nautical miles which is considerably greater than the distance from the U.S. to Australia. Global Hawk is designed to meet 
domestic needs including homeland security and has been demonstrated in drug interdiction. Global Hawks are also approved by the FAA to fly in U.S. airspace.

Another very successful UAV is the Predator which was also created in the mid-1990s but has since been enhanced with Hellfire missiles. "Named by Smithsonian's Air \& Space magazine as one of the top ten aircraft that changed the world, Predator is the most combat-proven Unmanned Aircraft System (UAS) in the world". The original version of the Predator, built by General Atomics, can fly at 25,000 feet for 40 hours at a maximum airspeed of 120 knots. In addition to missiles, the Predator can carry cameras, high resolution all weather radar and laser designators. The Predator is a little smaller than the Global Hawk but still has a wingspan of 55 feet.

At the very other extreme of size are the Micro Air Vehicles (MAVs) which are an interesting research focus area. There are many designs, some of which are bioinspired such as the flapping wing version shown in Figure (2.2).

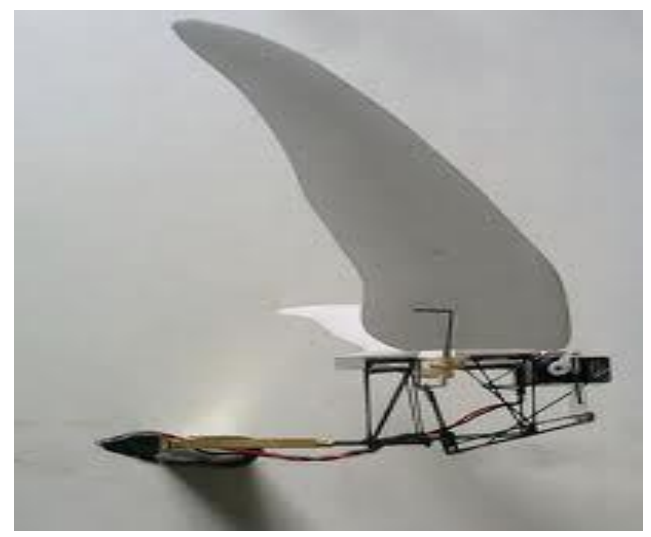

Figure 2.2 Micro Air vehicles

This design is being developed in Germany at the Biometrics-InnovationCentre and is inspired by a bird called the swift. Micro air vehicles are also modeled after various insects and generally use exotic designs and materials and are physically small. Additionally, although this design claims to be able to glide, the erratic motion caused by flapping wings could make this a difficult platform to operate a camera from. 
Although the designs in this class of UAV are fascinating, our interest was in attempting to produce a small UAV which could support a broad mission capability and these MAVs were dismissed as being too small. In addition to reviewing very large and very small UAVs, we were also intrigued by the requirements of DARPA's UAV forge competition which was posted. The UAV forge challenge uses crowd sourcing techniques to design and build a micro-UAV that can take off vertically, go to a designated distant location, monitor the location for up to three hours, identify specific objects and then return home. We found this challenge interesting because, since it was a DARPA research project, it represented pushing beyond the limits of what a small UAV had ever achieved.

The requirement for vertical liftoff also aligned with our thinking about the optimum form factor for a small UAV. Many of the deployed UAVs are fixed wing aircraft; however, we were looking for something more versatile that we believed could be built in small scale. The Quadcopter, like other helicopter designs, is able to take off without a runway, take video from a fixed hovering position, and finally maneuver through tight spaces as required. The Quadcopter also provides a superior payload capacity when compared to the helicopter and is a more stable platform. Since the Quadcopter was a vertical liftoff design, it aligned well with our team goals and therefore it became our baseline form factor.

\subsection{Project motivation}

We had a story to tell which explains, why we were motivated to take up this project. Once when one of our project members was watching television in which a news channel was broadcasting an accident in a factory which led to release of hazardous gases in the factory. The rescue personnel acting there cannot enter the factory due to hazardous gases and the management did not know whether some workers working in factory got stuck inside the factory. So, in the stated situation the rescue personnel cannot act for a rescue so 'when a man can't do the job then that man thinks of a machine to do it for him'. Therefore to solve the above situation we thought of an UAV to do the job for us in detecting the workers inside the factory 
using some heat detectors. The UAV which we planned to develop is Quadcopter because of some advantages said in above paragraphs.

In addition to the military uses and rescue operation uses of the small UAV, we were interested in evaluating applications in the commercial and industrial sector. Our premise was that if smaller and cheaper UAVs become readily available, new markets and uses will emerge. Potential new markets in commercial and industrial applications include inspecting pipelines or even inspecting dangerous areas like a meltdown site at a nuclear power plant. Disaster relief or crop assessment seems also to be likely areas where small UAVs could be useful. We were also motivated by on-campus uses such as monitoring parking or quick-look video of an incident, or monitoring hard to reach locations, or exploration of a collapsed building or other dangerous location. 
'Those who wish to succeed must ask the right preliminary questions'

-Aristotle

\section{CHAPTER 3}

\section{METHODOLOGY}

\subsection{Introduction}

This chapter will divide into two phases. The first phase understands the design process of quad copter and second is to understand its structure and its basic mathematical modeling.

\subsection{Flowchart}

Designs of Quadcopter are divided into two stages that is part design in first stage and full interface at second stage. Flow chart of Quadcopter design is described below: 


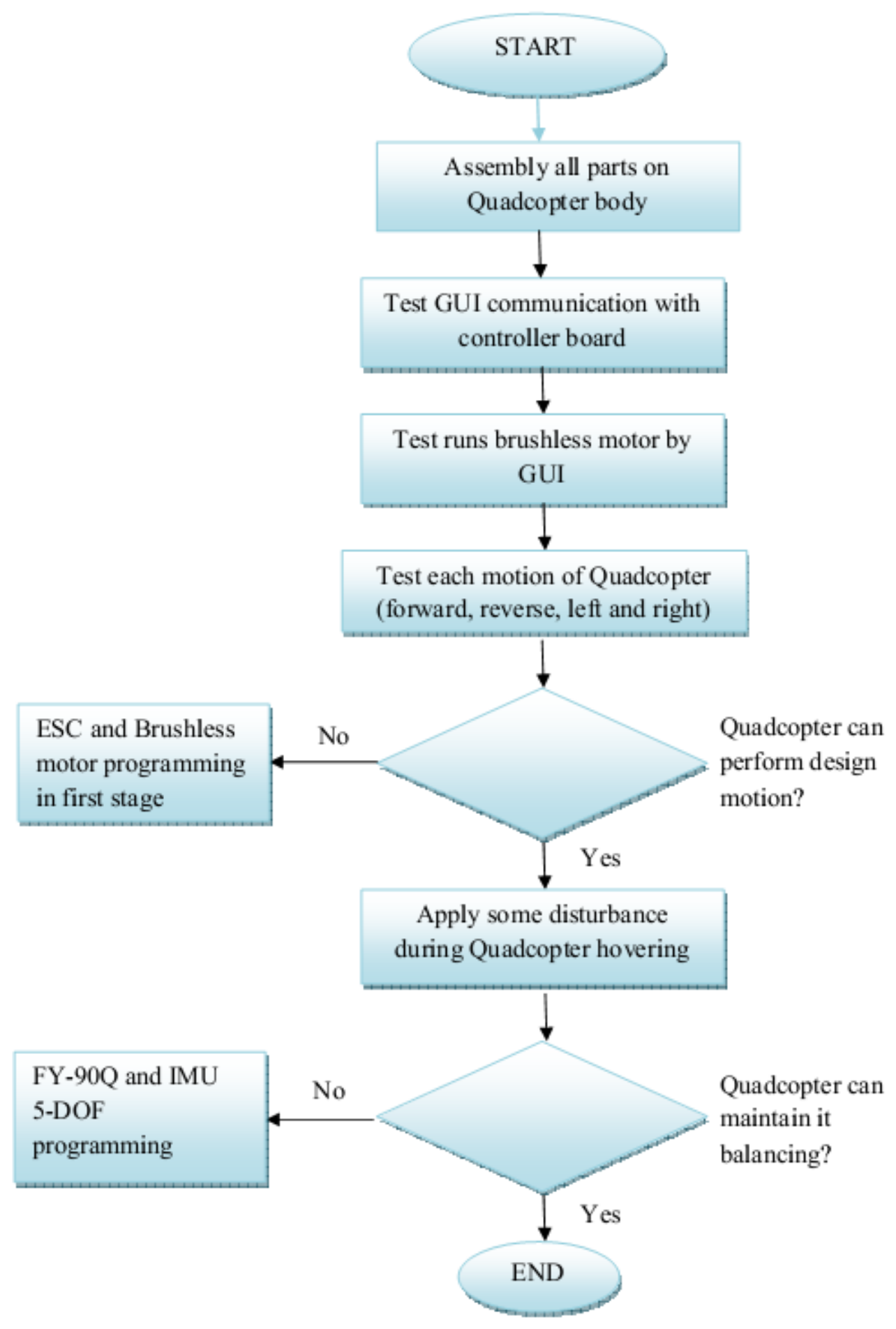

Figure 3.1: Flow chart of Quadcopter design

\subsection{Quadcopter Design constraints}

Quadcopter is an aerial vehicle consisting of four rotors connected in '+' or ' $\mathrm{X}$ ' design. Out of four rotors two should rotate in clockwise direction and the other two in 
anti-clockwise rotation. The four propellers are not identical. One set of propellers (Clockwise rotation) are tilted towards right and the other set (Anti-clockwise rotation) are tilted towards left.

The reason for this is that the motor torque and the law of physics will make the Quadcopter spin around itself if all the propellers were rotating the same way, without any chance of stabilizing it. By making the propeller pairs spin in each direction, but also having opposite tilting, all of them will provide lifting thrust without spinning in the same direction. This makes it possible for the Quadcopter to stabilize the yaw rotation, which is the rotation around itself.

\subsubsection{Frame constraints}

We have two types of frame configurations the ' $\mathrm{X}$ ' and the ' + '.

The $\mathrm{X}$ and + configurations are as follows:

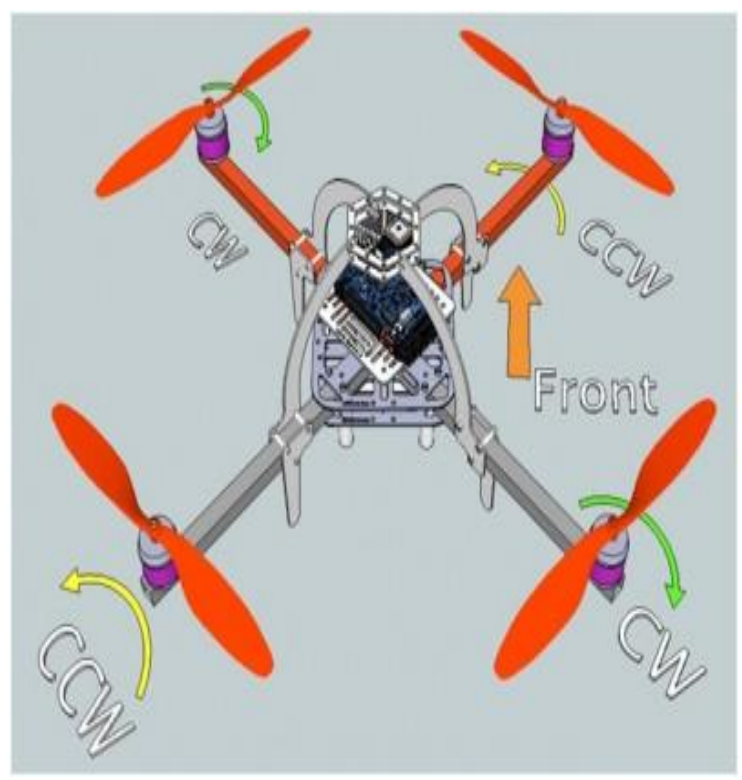

Figure 3.2 Quadcopter X configuration

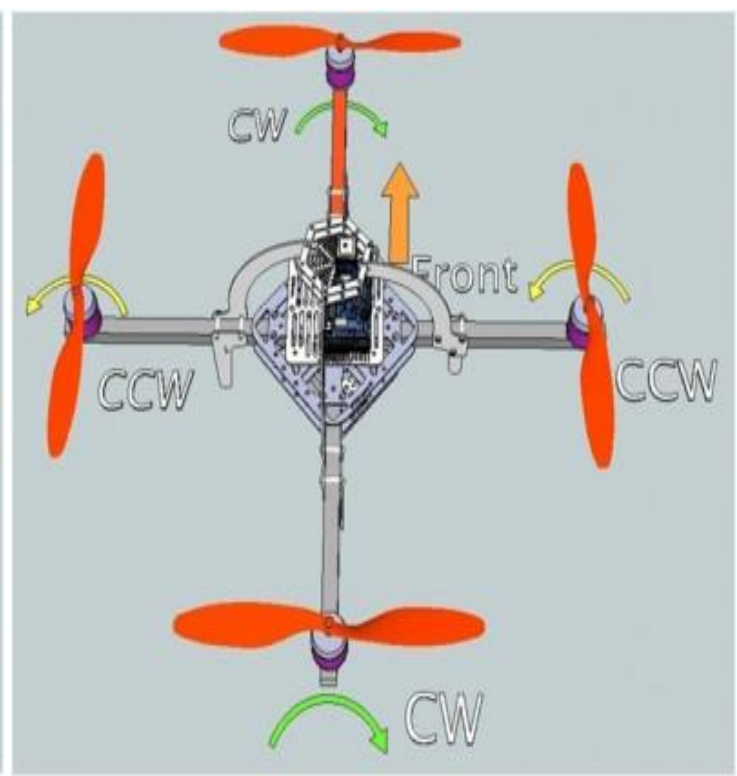

Figure 3.3 Quadcopter + configuration

The difference is how the motors have to be controlled. For the + configuration the motor controlling is fairly simple as you just have one motor assigned to each direction. The negative aspect of the + configuration though is that you only have a single motor to provide extra thrust (speed up) when you want to move to another 
direction. In the $\mathrm{X}$ configuration you will always have two motors working together on changing direction.

On basing of above advantage we are moving forward with the $\mathrm{X}$ configuration.

\subsection{Quadcopter movement mechanism}

Quadcopter can described as a small vehicle with four propellers attached to rotor located at the cross frame. This aim for fixed pitch rotors are use to control the vehicle motion. The speeds of these four rotors are independent. By independent, pitch, roll and yaw attitude of the vehicle can be control easily. Pitch, roll and yaw attitude off Quadcopter are shown in Figure 3.2, 3.3 and 3.4.

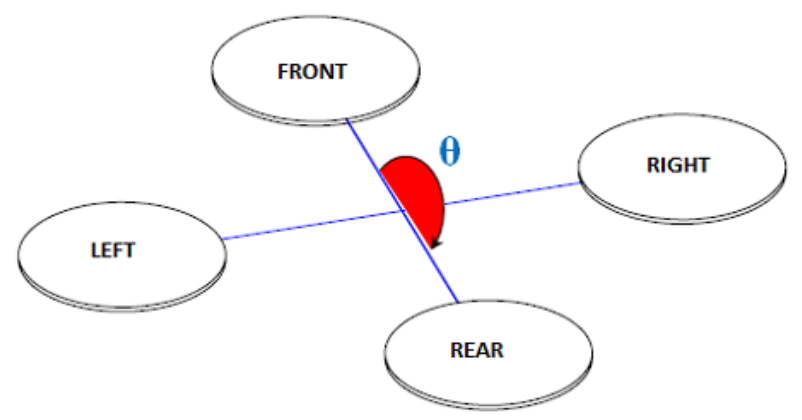

Figure 3.4: Pitch direction of Quadcopter

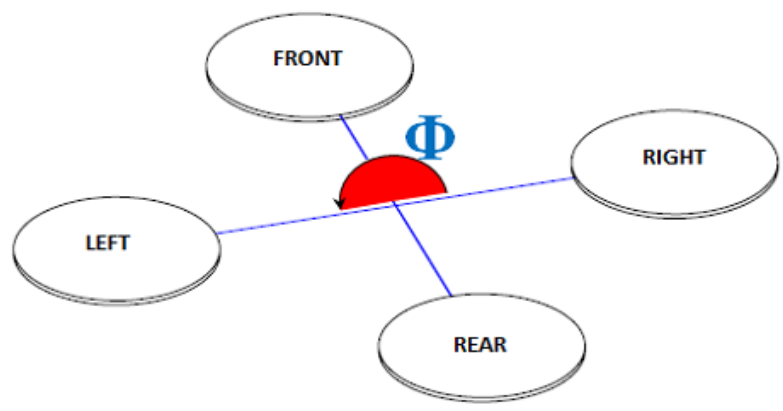

Figure 3.5: Roll direction of Quadcopter 


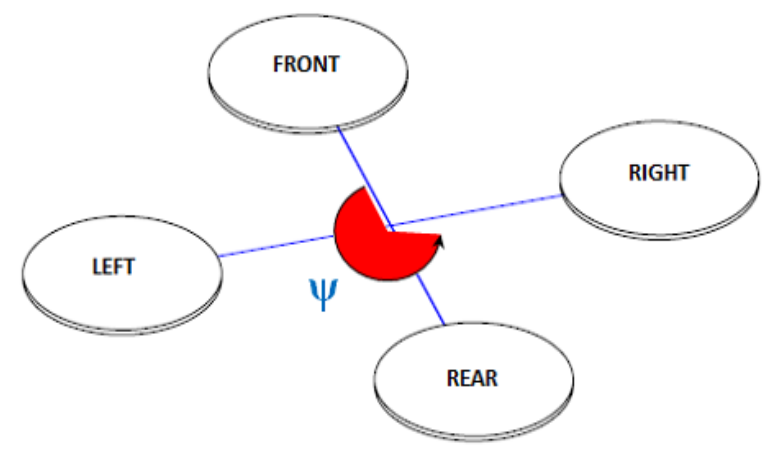

Figure 3.6 Yaw direction of Quadcopter

Quadcopter have four inputs force and basically the thrust is produced by the propeller that connect to the rotor. The motion of Quadcopter can control through fix the thrust that produced. These thrust can control by the speed of each rotor.

\subsubsection{Take-off and landing motion mechanism}

Take-off is movement of Quadcopter that lift up from ground to hover position and landing position is versa of take-off position. Take-off (landing) motion is control by increasing (decreasing) speed of four rotors simultaneously which means changing the vertical motion. Figure 3.7 and 3.8 illustrated the takeoff and landing motion of Quadcopter respectively.

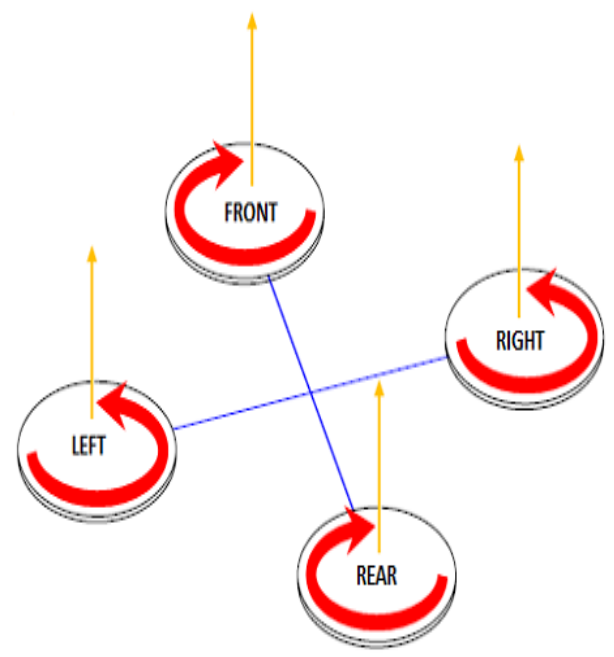

Figure 3.7 Take-off motion

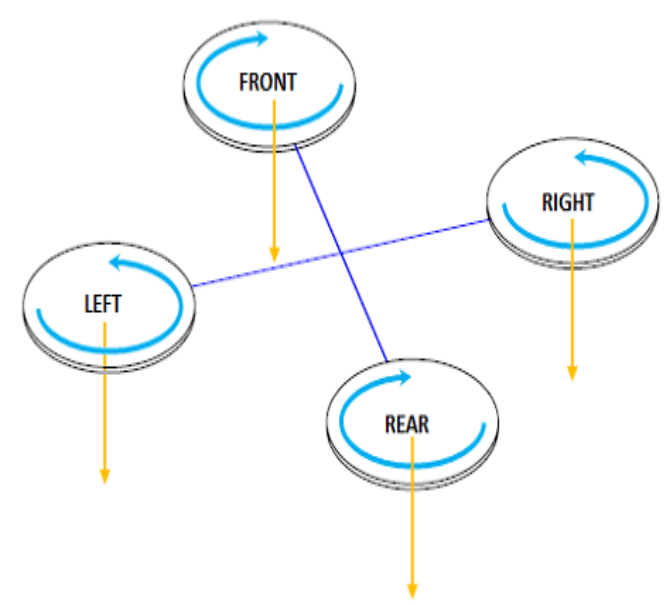

Figure 3.8 Landing motion 


\subsubsection{Forward and Backward motion}

Forward (backward) motion is control by increasing (decreasing) speed of rear (front) rotors and Decreasing (increasing) front (rear) rotors speed simultaneously, will also affect the pitch angle of the Quadcopter. The forward and backward motions of Quadcopter are represented in Figure 3.9 and 3.10 respectively.

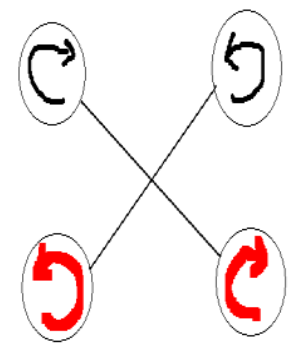

Figure 3.9 Forward motion

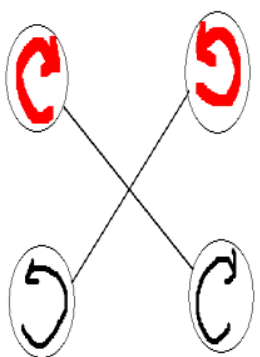

Figure 3.10 Backward motion

\subsubsection{Left and right motion}

For left and right motion, it can control by changing the yaw angle of Quadcopter. Yaw angle can control by increasing (decreasing) counter-clockwise rotors speed while decreasing (increasing) clockwise rotor speed. Figure 3.11 and 3.12 show the right and left motion of Quadcopter.

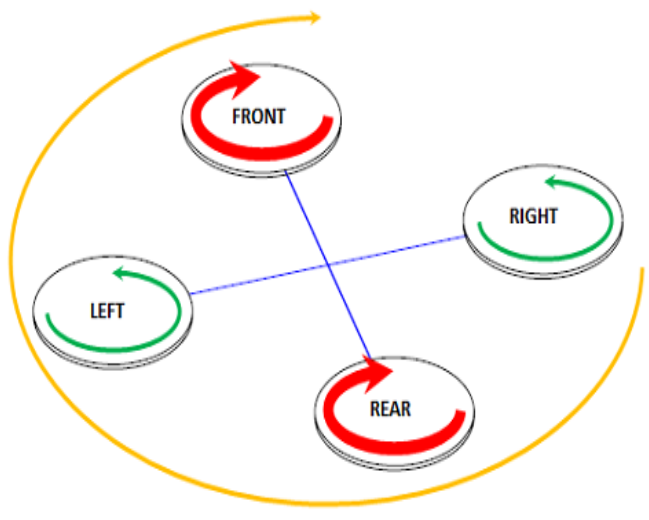

Figure 3.11 Right motion

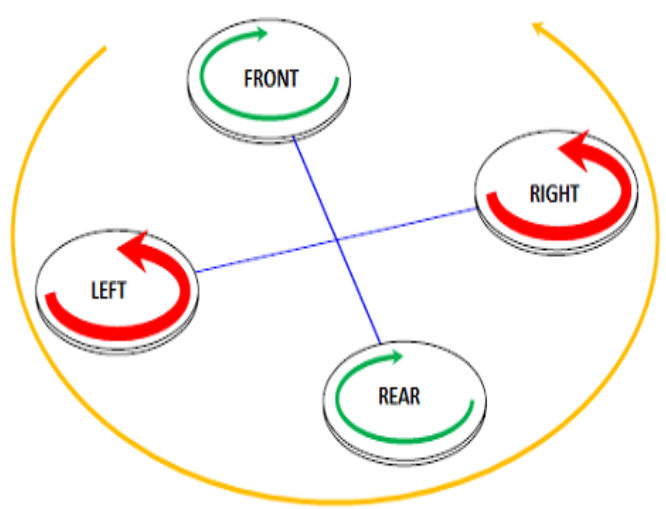

Figure 3.12 Left motion 


\subsubsection{Roll right or roll left}

Roll right (left) motion is control by increasing (decreasing) speed of left (right) rotors and decreasing (increasing) right (left) rotors speed simultaneously, will also affect the roll angle of the Quadcopter. The roll right and left motions of Quadcopter are represented in Figure 3.13 and 3.14 respectively.

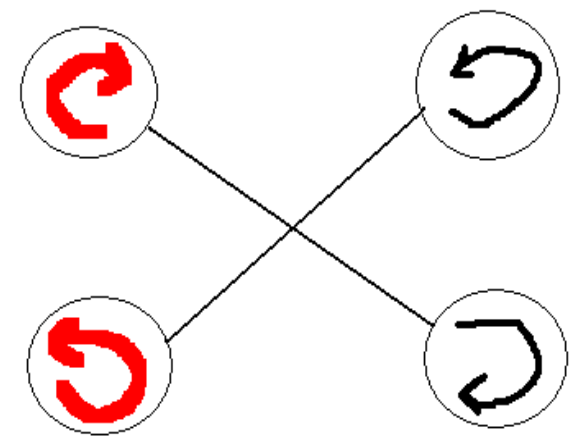

Figure 3.13 Roll right

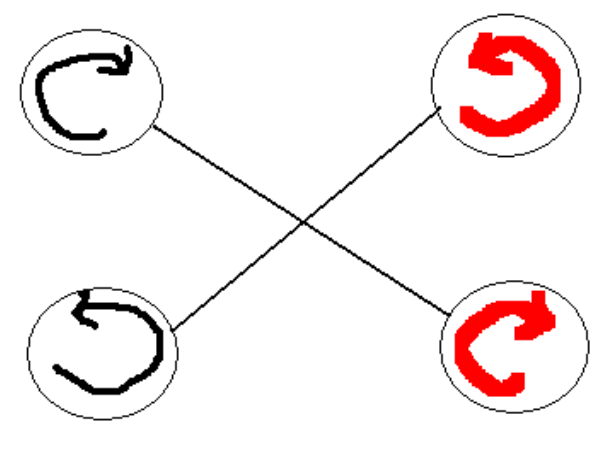

Figure 3.14 Roll left

\subsubsection{Hovering or static position}

The hovering or static position of Quadcopter is done by two pairs of rotors are rotating in clockwise and counter-clockwise respectively with same speed. By two rotors rotating in clockwise and counter-clockwise position, the total sum of reaction torque is zero and this allowed Quadcopter in hovering position.

\subsection{Quadcopter mathematical modeling}

The schematic movement of Quadcopter is represented in Figure 3.15 and based on this schematic, the Quadcopter mathematical modeling is derived as below: 


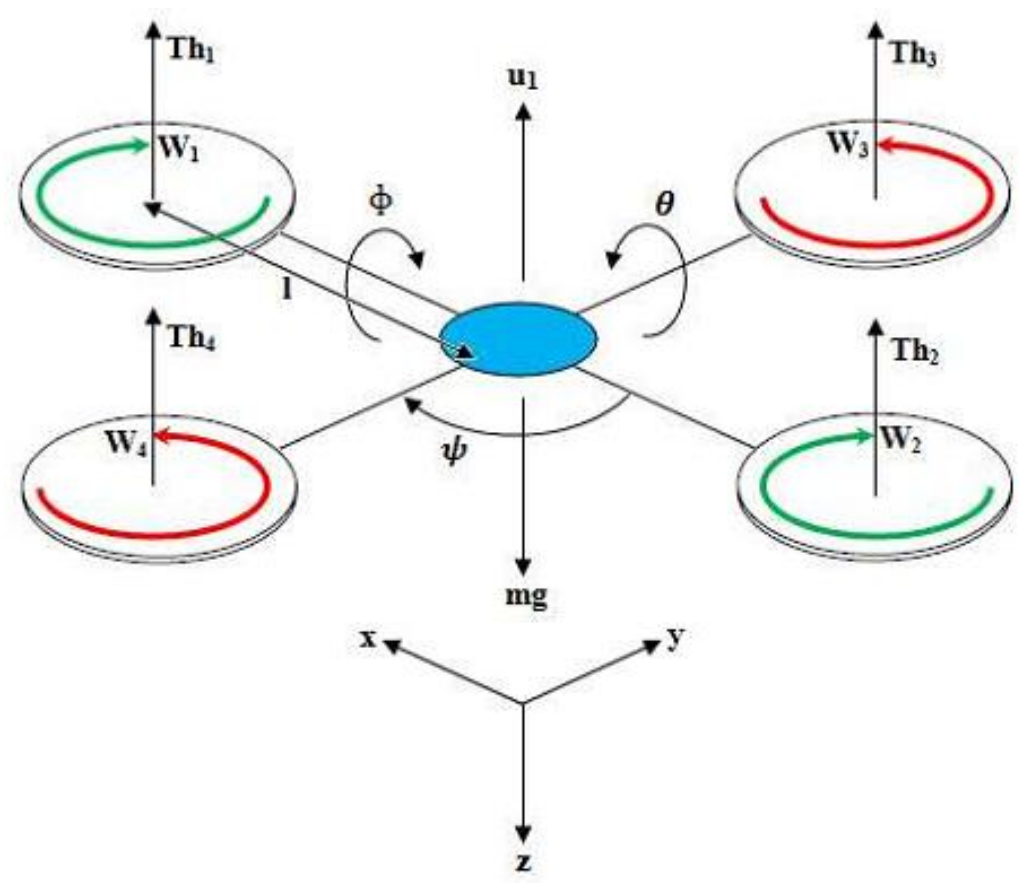

Figure 3.15 Schematic of Quadcopter

Where,

$\mathrm{U} 1=$ sum of the thrust of each motor

Th1 $=$ thrust generated by front motor

Th2 $=$ thrust generated by rear motor

Th3= thrust generated by right motor

Th4= thrust generated by left motor

$\mathrm{m}=$ mass of Quadcopter

$\mathrm{g}=$ the acceleration of gravity

$1=$ the half length of the Quadcopter

$\mathrm{x}, \mathrm{y}, \mathrm{z}=$ three position

$\theta, \Phi, \psi=$ three Euler angles representing pitch, roll, and yaw

The dynamics formulation of Quadcopter moving from landing position to a fixed point in the space is given as: 


$$
R x y z=\left[\begin{array}{ccc}
C_{\phi} C_{\theta} & C_{\phi} S_{\theta} S_{\psi}-S_{\phi} C_{\psi} & C_{\phi} S_{\theta} C_{\psi}+S_{\phi} S_{\psi} \\
C_{\phi} S_{\theta} & S_{\phi} S_{\theta} S_{\psi}+C_{\phi} C_{\psi} & S_{\phi} S_{\phi} C_{\psi}-C_{\phi} S_{\psi} \\
-S_{\theta} & C_{\theta} S_{\psi} & C_{\theta} C_{\psi}
\end{array}\right]
$$

Where,

$$
\begin{aligned}
& \mathrm{R}=\text { matrix transformation } \\
& S_{\theta}=\operatorname{Sin}(\theta), S_{\phi}=\operatorname{Sin}(\phi), S_{\psi}=\operatorname{Sin}(\psi) \\
& C_{\theta}=\operatorname{Cos}(\theta), C_{\phi}=\operatorname{Cos}(\phi), C_{\psi}=\operatorname{Cos}(\psi)
\end{aligned}
$$

By applying the force and moment balance laws, the Quadcopter motion equation are given in Equation (3.2) till (3.4) and Pythagoras theorem is computed as Figure 3.16.

$$
\begin{aligned}
& \ddot{x}=\mathrm{u}_{1}(\operatorname{Cos} \phi \operatorname{Sin} \theta \operatorname{Cos} \psi+\operatorname{Sin} \phi \operatorname{Sin})-\mathrm{K}_{1} \dot{\mathrm{x}} / \mathrm{m} \\
& \ddot{y}=\mathrm{u}_{1}(\operatorname{Sin} \phi \operatorname{Sin} \theta \operatorname{Cos} \psi+\operatorname{Cos} \phi \operatorname{Sin})-\mathrm{K}_{2} \dot{\mathrm{y}} / \mathrm{m} \\
& \ddot{z}=\mathrm{u}_{1}(\operatorname{Cos} \phi \operatorname{Cos} \psi)-\mathrm{g}-\mathrm{K}_{3} \dot{z} / \mathrm{m}
\end{aligned}
$$

Where,

$\mathrm{Ki}=$ drag coefficient (Assume zero since drag is negligible at low speed)

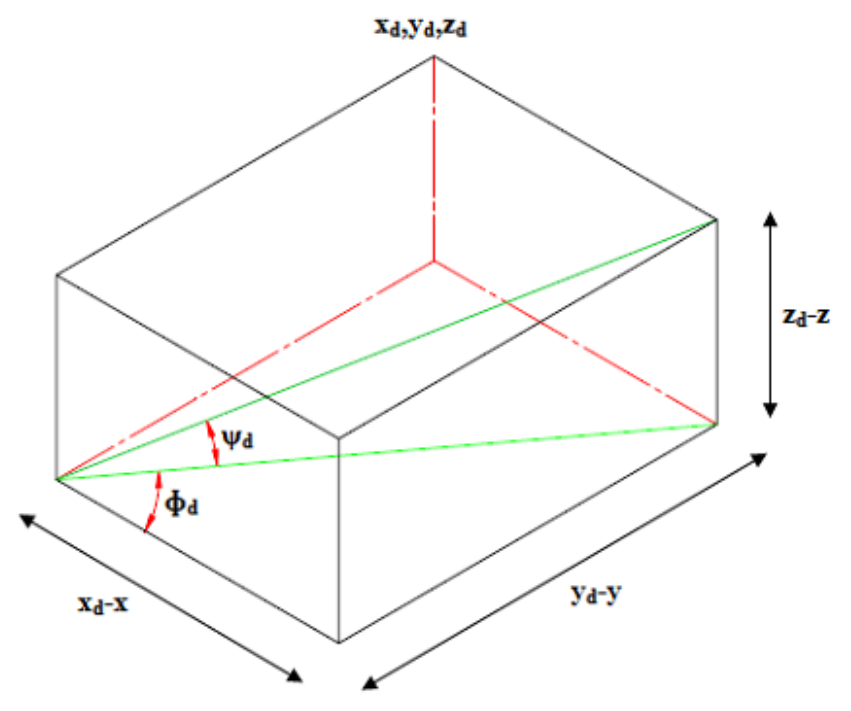

Figure 3.16 Angle Movement of Quadcopter

The angle $\Phi_{\mathrm{d}}$ and $\psi_{\mathrm{d}}$ in Figure 3.16 are determined using Equation (3.5) and (3.6) respectively. 


$$
\begin{aligned}
\phi_{\mathrm{d}} & =\tan ^{-1}\left(\frac{y d-y}{x d-x}\right) \\
\psi_{\mathrm{d}} & =\tan ^{-1}\left(\frac{z d-z}{\sqrt{(x d-x)^{2}+(y d-y)^{2}}}\right)
\end{aligned}
$$

Quadcopter have four controller input forces U1, U2, U3, and U4 that will affects certain side of Quadcopter. U1 affect the attitude of the Quadcopter, U2 affects the rotation in roll angle, $\mathrm{U} 3$ affects the pitch angle and $\mathrm{U} 4$ control the yaw angle. To control the Quadcopter movement is done by controlling each input variable. The equations of them are as below:

$$
\mathrm{U}\left\{\begin{aligned}
\mathrm{U}_{1} & =\left(\mathrm{Th}_{1}+\mathrm{Th}_{2}+\mathrm{Th}_{3}+\mathrm{Th}_{4}\right) / \mathrm{m} \\
\mathrm{U}_{2} & =1\left(-\mathrm{Th}_{1}-\mathrm{Th}_{2}+\mathrm{Th}_{3}+\mathrm{Th}_{4}\right) / \mathrm{I}_{1} \\
\mathrm{U}_{3} & =1\left(-\mathrm{Th}_{1}+\mathrm{Th}_{2}+\mathrm{Th}_{3}-\mathrm{Th}_{4}\right) / \mathrm{I}_{2} \\
\mathrm{U}_{4} & =1\left(\mathrm{Th}_{1}+\mathrm{Th}_{2}+\mathrm{Th}_{3}+\mathrm{Th}_{4}\right) / \mathrm{I}_{3}
\end{aligned}\right.
$$

Where,

$\mathrm{Th}_{\mathrm{i}}=$ thrust generated by four motor

$\mathrm{C}=$ the force to moment scaling factor

$I_{i}=$ the moment of inertia with respect to the axes

Then the second derivatives of each angle are:

$$
\begin{aligned}
& \ddot{\theta}=\mathrm{U}_{2}-1 \mathrm{~K}_{4} \dot{\theta} / \mathrm{I}_{1} \\
& \ddot{\psi}=\mathrm{U}_{3}-1 \mathrm{~K}_{5} \dot{\psi} / \mathrm{I}_{2} \\
& \ddot{\phi}=\mathrm{U}_{1}-1 \mathrm{~K}_{6} \dot{\phi} / \mathrm{I}_{3}
\end{aligned}
$$




\section{CHAPTER 4}

\section{TECHNICAL SPECIFICATIONS}

\subsection{Brushless DC Motors}

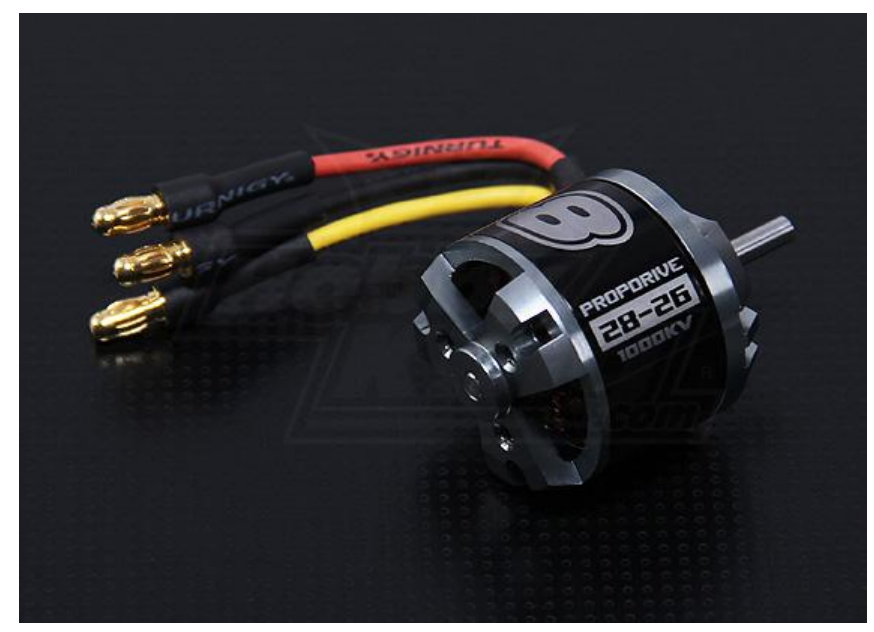

Figure 4.1

Model: $\quad$ NTM Prop Drive Series $28261000 \mathrm{kv}$

Kv:

$1000 \mathrm{rpm} / \mathrm{v}$

Max current: 21A

Max Power: 200W @ 11.1V (3S) / 315W @ 15V (4S)

Shaft: $\quad 3 \mathrm{~mm}$

Weight: $\quad 54 \mathrm{~g}$

ESC: $\quad 20 \sim 30 \mathrm{~A}$

Cell count: $\quad 3 s \sim 4$ s Lipoly

Bolt holes: $16 \mathrm{~mm} \mathrm{\&} 19 \mathrm{~mm}$ 
Bolt thread: $\quad$ M3

Connection: $\quad 3.5 \mathrm{~mm}$ Bullet-connector

Brushless motors are a bit similar to normal DC motors in the way that coils and magnets are used to drive the shaft. Though the brushless motors do not have a brush on the shaft which takes care of switching the power direction in the coils, and this is why they are called brushless.

Instead the brushless motors have three coils on the inner (center) of the motor, which is fixed to the mounting. On the outer side it contains a number of magnets mounted to a cylinder that is attached to the rotating shaft. So the coils are fixed which means wires can go directly to them and therefore there is no need for a brush.

The reason why Quadcopter use brushless motors instead of normal DC motors is the much higher speeds and less power usage for the same speed. The brushless motors are more efficient as there is no power lost as there is in the brush-transition on the DC motors.
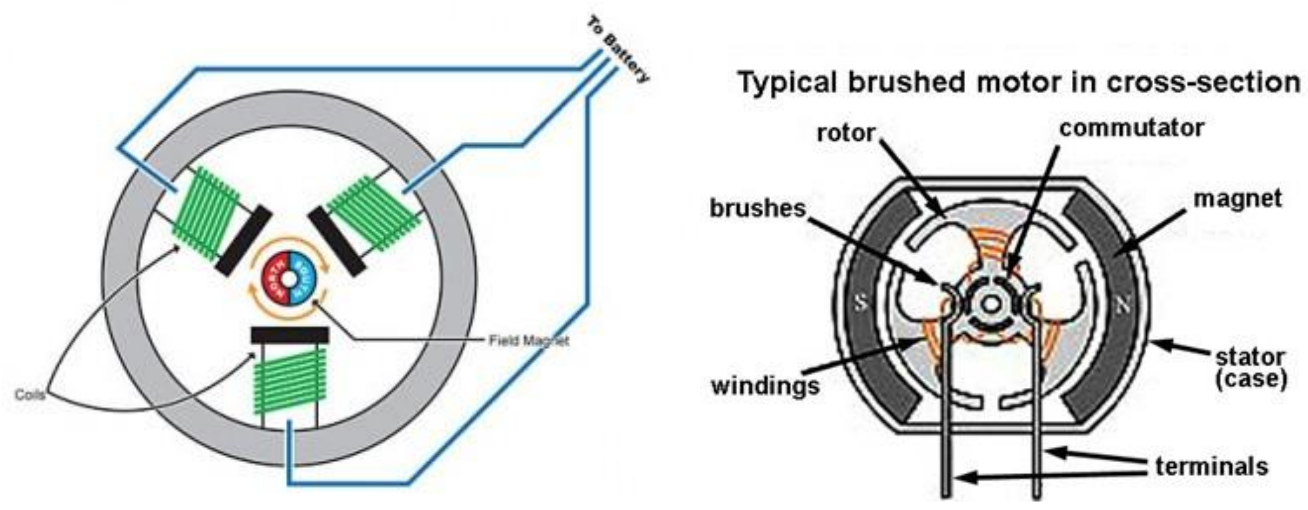

Figure 4.2 Brushless and brushed motor internal. 


\subsection{Propellers}

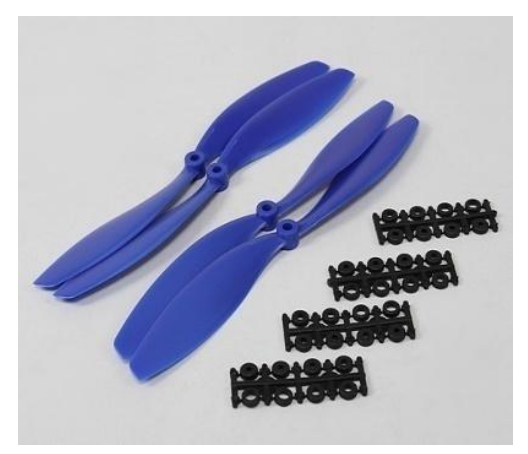

Figure 4.3 Propellers

Type: $\quad$ Pusher and Puller pair of propellers

Diameter: 10 Inches

Pitch: $\quad 4.5$ Inches

Weight: $\quad 13 \mathrm{gms}$

Climb rate: $\quad 12.48 \mathrm{~m} / \mathrm{sec}$

Generally propellers are in slightly unbalanced and due to the friction free rotation of this unit a small weight in balance becomes very obvious. So to attain balance, there are two methods namely static balancing and dynamic balancing.

A propeller can be statically balanced only by removing it from the aircraft and evaluating the balance on a special fixture and then weights are added or removed from the propeller assembly.

Dynamic Balancing use sensors to provide data to a digital processor during brief engine runs at 2000-2400rpm. This balancing is done to provide for the lowest level of vibration in its operating range. But it takes approximately $2 \mathrm{hrs} / \mathrm{engine.}$ 


\subsection{Electronic speed Controllers (ESC)}

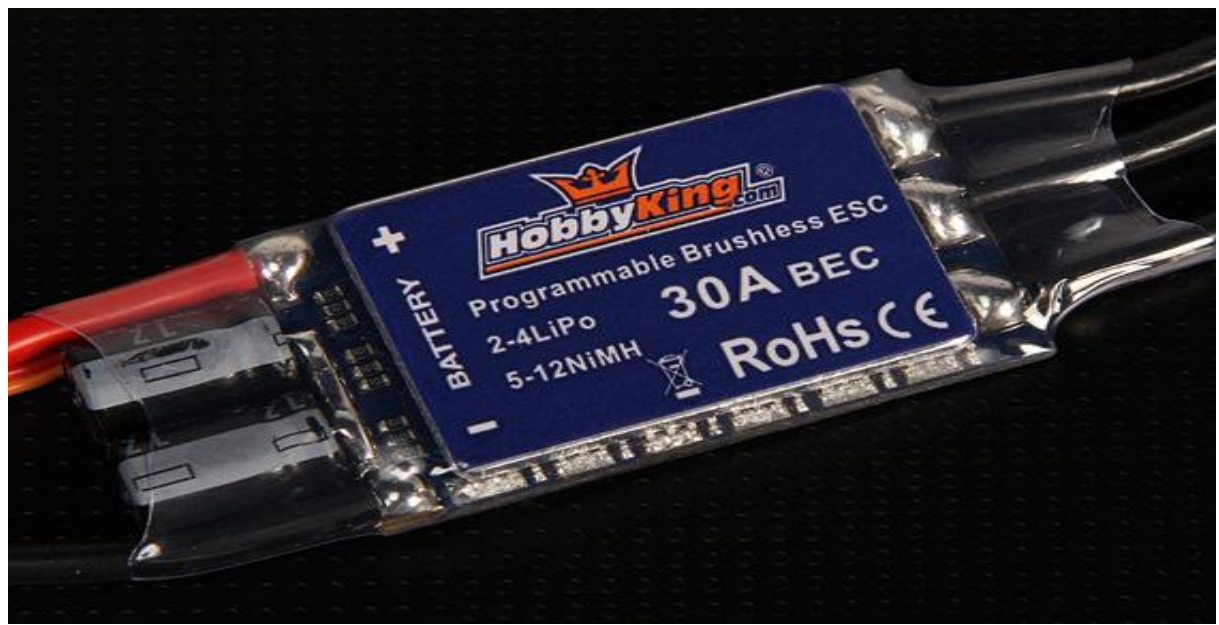

Figure 4.4 ESC

Model:

30A Blue Series Brushless Speed Controller

Continuous Current: $30 \mathrm{~A}$

Burst Current: $\quad 40 \mathrm{~A}$

Battery:

2-4 Cell Lipo / 5-12 Cell Ni-XX

SBEC:

5V/3A Output

Size:

$23 * 43 * 6 \mathrm{~mm}$

Weight: $\quad 28 \mathrm{~g}$

As the brushless motors are multi-phased, normally 3 phases, you can't just apply power to it to make it spin. The motors requires some special phase-control electronics that is capable of generating three high frequency signals with different but controllable phases, but the electronics should also be able to source a lot of current as the motors can be very power-hungry.

In this case we got the Electronic Speed Controllers, known as ESCs. The ESCs is simply a brushless motor controller board with battery input and a three phase output for the motors. For the control it is usually just a simple PPM signal (similar to PWM) that ranges from $1 \mathrm{~ms}$ (min speed=turn off) to $2 \mathrm{~ms}$ (max speed) in pulse width. The frequency of the signals does also vary a lot from controller to controller, but for a Quadcopter it is recommended to get a controller that supports at least $200 \mathrm{~Hz}$ or even better $300 \mathrm{~Hz}$ PPM signal, as it should be possible to change the motor speeds 
very quickly to adjust the Quadcopter to the stable position. It is also possible to get ESCs that is controlled through One Wire of I2C. These tends to be much more expensive though, but sometimes it is also possible to mod other ESCs to add the I2C feature.

\subsection{Battery}

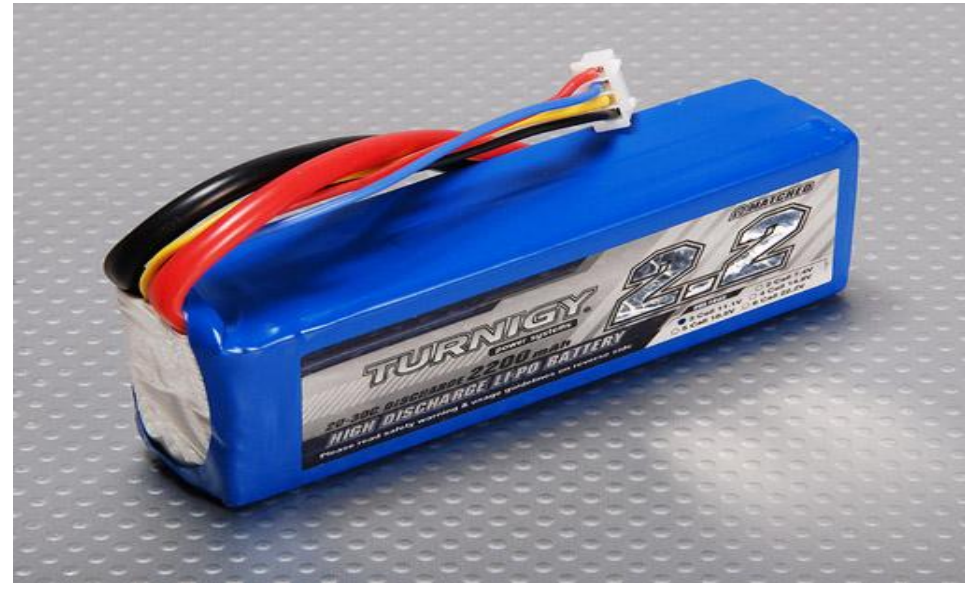

Figure 4.5 Battery

Minimum Capacity: $\quad 2200 \mathrm{mAh}$

Configuration: $\quad 3 \mathrm{~S} 1 \mathrm{P} / 11.1 \mathrm{v} / 3 \mathrm{Cell}$

Constant Discharge: $\quad 20 \mathrm{C}$

Peak Discharge (10sec): $30 \mathrm{C}$

Pack Weight: $\quad 185 \mathrm{~g}$

Pack Size: $\quad 103 \times 33$ × $\quad 24 \mathrm{~mm}$

Charge Plug: $\quad$ JST-XH

Discharge Plug: $\quad$ XT60

All this leads to the battery, the power source for the whole device. For the battery two types can be used, where of one of them is highly recommended.The NiMH and the LiPo. We won't say much about the NiMH as most communities tells us to stay away from these for driving Quadcopter as they first and foremost are not able to provide enough current and secondly they weight a lot more than LiPo batteries when they have the necessary current ratings. Instead we should talk about LiPo batteries, but in this world there are also a lot of different variants of these too. 
LiPo batteries can be found in packs of everything from a single cell (3.7V) to over 10 cells $(37 \mathrm{~V})$. The cells are usually connected in series, making the voltage higher but giving the same amount of amp-hours.

For a Quadcopter you should go after the 3SP1 batteries which means 3 cells connected in series as 1 parallel (just forget the parallel, as it has no sense because we just use 3 cells in series). This should give us $11.1 \mathrm{~V}$ but at fully charged it actually gives us around $12 \mathrm{~V}$ instead.

For a brushless motor with a Kv-rating of 1000 , this gives us a maximum of 12000 rounds per minute. This number is totally fictive as the battery voltage will drop immediately to around $11.1 \mathrm{~V}$ (at fully charged state) when current is being drained. Anyways, this gives us a good idea about how fast the propellers will be spinning!

As for the battery capacity regards you should make some calculations on how much power your motors will draw and then decide how long flight time you want and how much influence the battery weight should have on the total weight. A good rule of thumb is that you with four EPP1045 propellers and four $\mathrm{Kv}=1000$ rated motor will get the number of minutes of full throttle flight time as the same number of amphours in your battery capacity. This means that if you have a 4000mAh battery, you will get around 4 minutes of full throttle flight time though with a $1 \mathrm{KG}$ total weight you will get around 16 minutes of hover.

Another thing to be-aware of when selecting the right battery is the discharge rate, formerly known as the $\mathrm{C}$-value. The $\mathrm{C}$-value together with the battery capacity indicates how much current you are able to source from the battery. The calculations follow this simple rule: MaxSource $=$ DischargeRate $\mathrm{x}$ Capacity 


\subsection{Battery Charger}

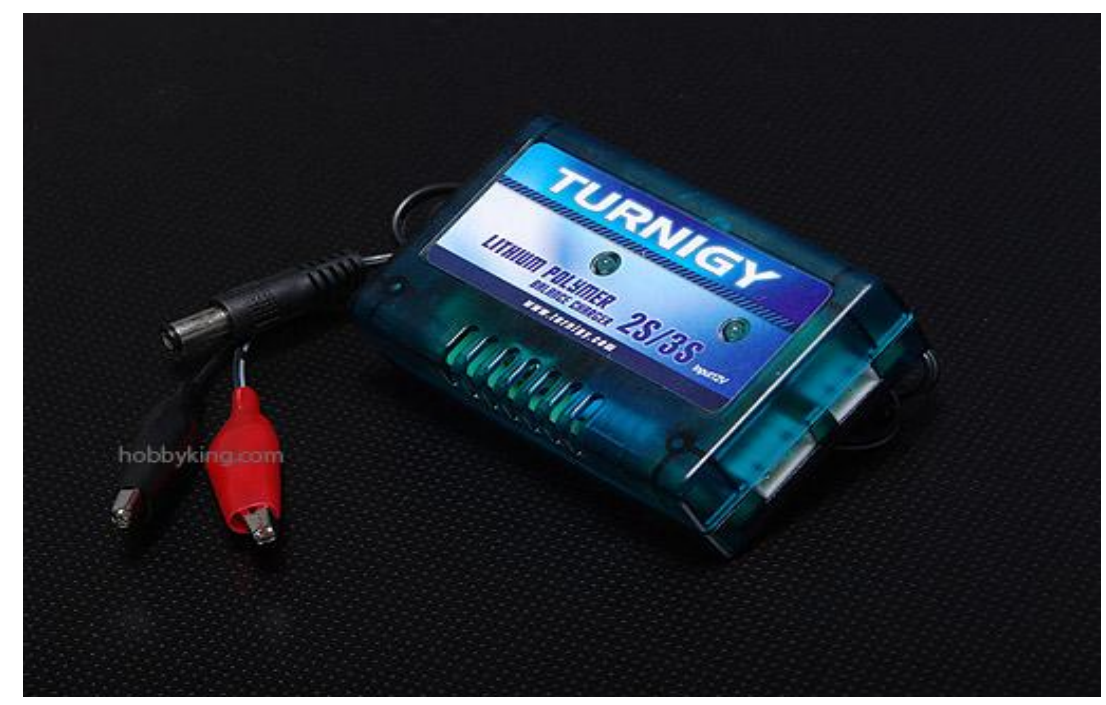

Figure 4.6 Battery charger

Input: $\quad 12-15 v \mathrm{DC}$

- Turnigy balancer \& Charger $2 \mathrm{~S}-3 \mathrm{~S}$

- charges at a rate of around $800 \mathrm{mAh}$.

- There are two LED status lights. The red light indicates that power is connected to the charger. The green light means that charging is in progress. The green light goes out when charging is complete.

\subsection{Voltage Detector}

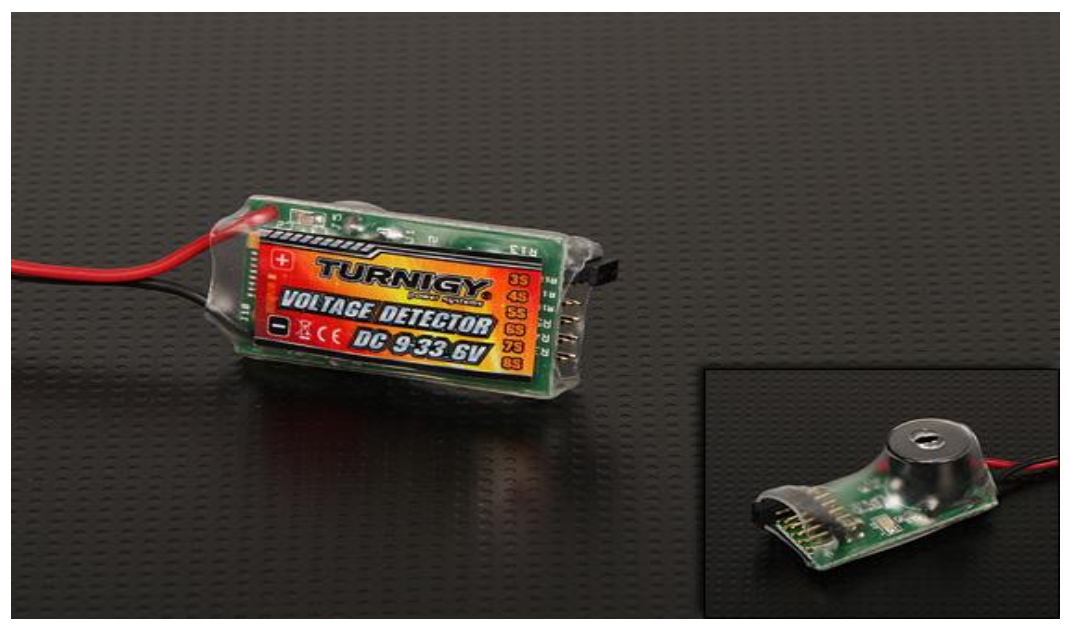

Figure 4.7 Voltage detector 
Operating Voltage: $\quad$ 9 33.6V

Weight:

$6 \mathrm{~g}$

- The 3 8S voltage detector will give a warning buzzer as well as LED indication for High, Average, below average, and Low voltages on your Lipoly battery.

- The buzzer is also quiet loud. Its varied warning range is great for those with gliders that need time to bring the plane down from a high altitude.

- Turnigy voltage detector has polarity protection.

\subsection{Transmitter \& Receiver}
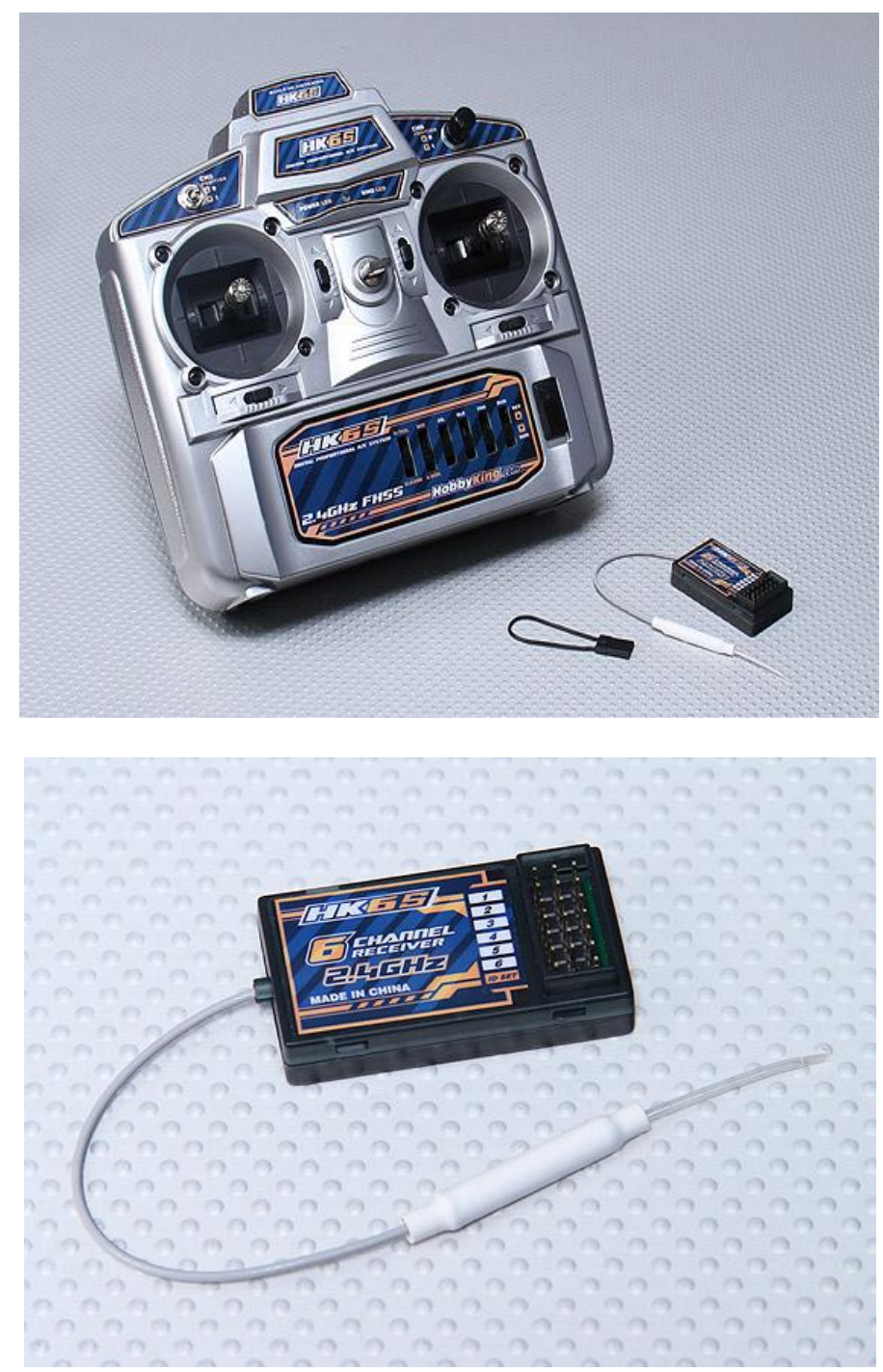

Figure $4.8 \mathrm{Tx}$ and $\mathrm{Rx}$

- 6 channel $2.4 \mathrm{GHz}$ transmitter. 
- Features build-in antenna.

- Easy to use control for basic models.

- FHSS 2.4ghz Technology

- Includes 6-channel receiver

- $\quad$ Mode 2 (THROTTLE ON THE LEFT)

How to control vehicle with this TX???

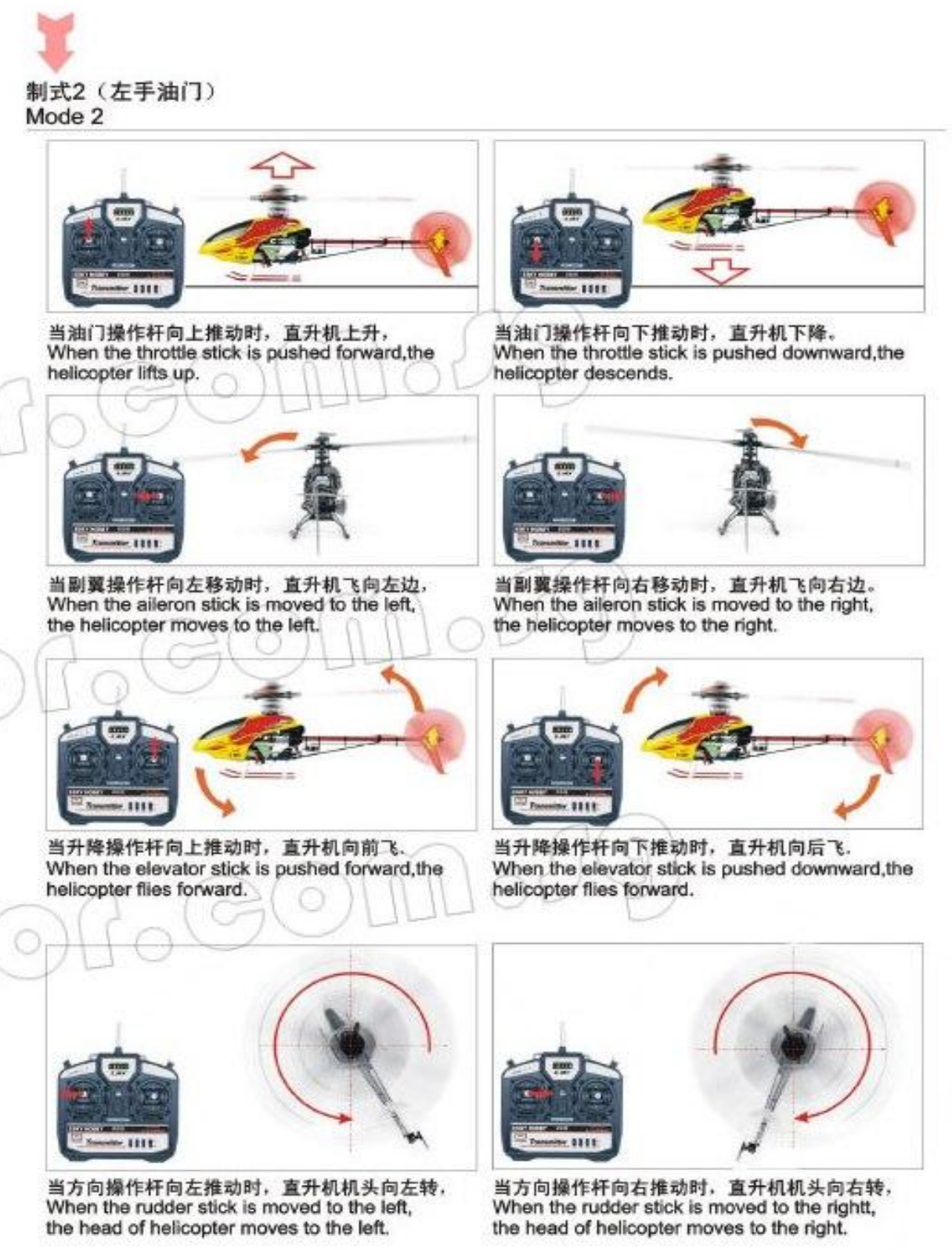

Figure 4.9 How to control?

This control is shown for helicopter in the above figure but we can apply the same to quadcopter too. 


\subsection{CCD Camera \& Tx-Rx}

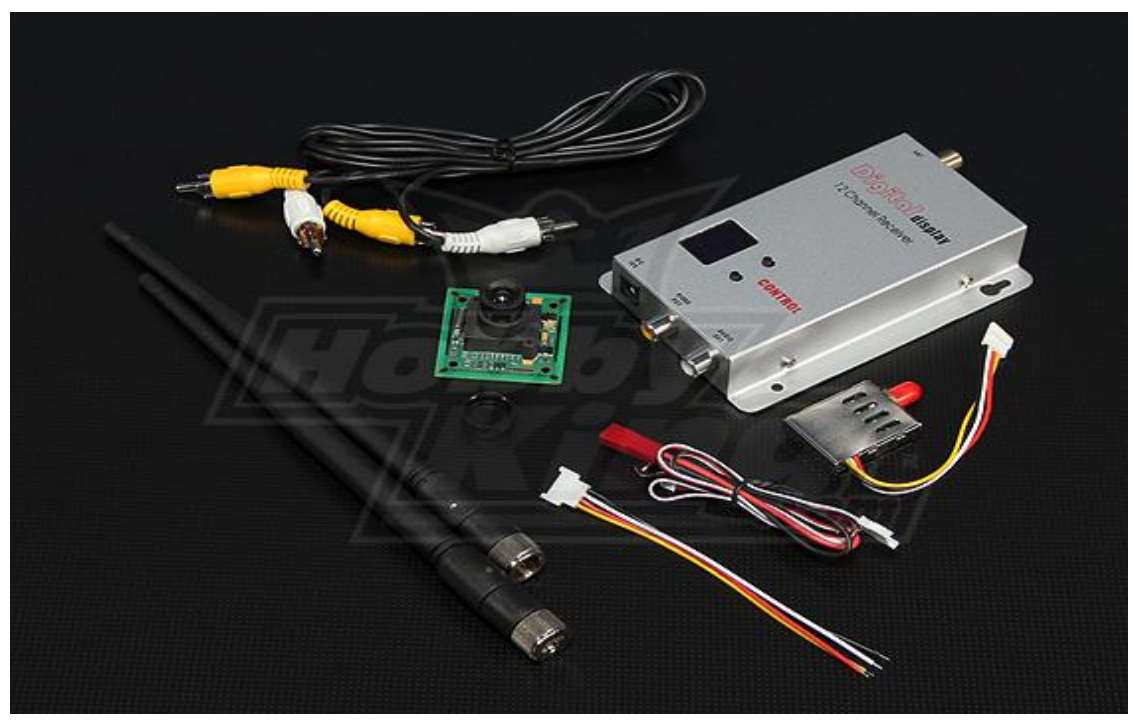

Figure 4.10 Camera and Tx-Rx

Image device:

Pixel:

Horizontal resolution:

White balance:

Lens:

Input voltage:

Electric current:

Electronic Shutter:

Video output:

Operation Temp.:

Size:

Power supply: 1/3-inch Sony super HAD color CCD

$752 \times 582$ (NTSC)

520TV Lines

Auto Tracking White Balance

3.6-6mm

DC12V

$80 \mathrm{MA} \pm 5 \mathrm{MA}$

Auto

$1.0 \mathrm{Vp} \sim \mathrm{p} 75 \Omega$

$-10 \sim+50$

$38 \times 38 \mathrm{~mm}$

$12 \mathrm{~V} / 150 \mathrm{~mA}$

FPV Tx Specifications:

Channel: 4-Ch, AV synchronization

Power: $\quad 200 \mathrm{~mW}$

Input voltage: $12 \mathrm{~V}$

Weight: $\quad 18 \mathrm{~g}$ 
Size:

$31 \times 88 \times 8 \mathrm{~mm}$

Frequency: $\quad 0.9 \mathrm{G}, 0.910 \mathrm{G}, 0.980 \mathrm{G}, 1.010 \mathrm{G}, 1.040 \mathrm{G}$

\subsection{Flight Control Board}

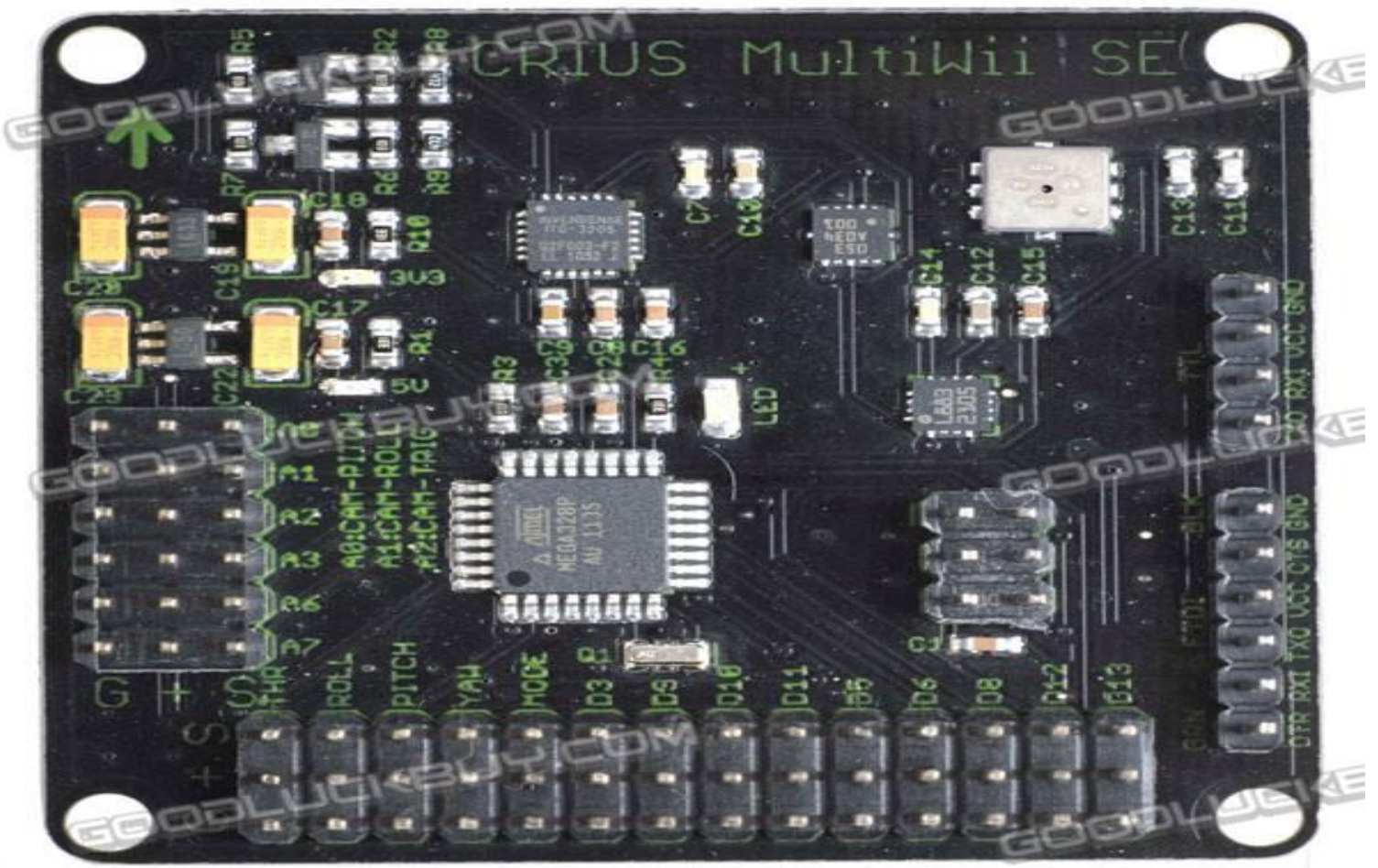

Figure 4.11 Crius Multiwii SE QuadX Flight Control Board

- ATMEGA328P microcontroller

- ITG3205 three-axis digital gyroscope

- BMA180 triaxial accelerometer

- BMP085 pressure sensor

- HMC5883L axis magneto resistive sensor (electronic compass)

- Dimensions $50 \mathrm{~mm}$ X $50 \mathrm{~mm}$

- Standard Mk style mounting holes $45 \mathrm{~mm}$ X $45 \mathrm{~mm}$

- Weigh $13.9 \mathrm{~g}$

- Separate 3.3v regulated supply for the sensors

- On board logic level converter

- All the out puts are labeled for easy identification 


\subsubsection{ATMEGA 328P Microcontroller}

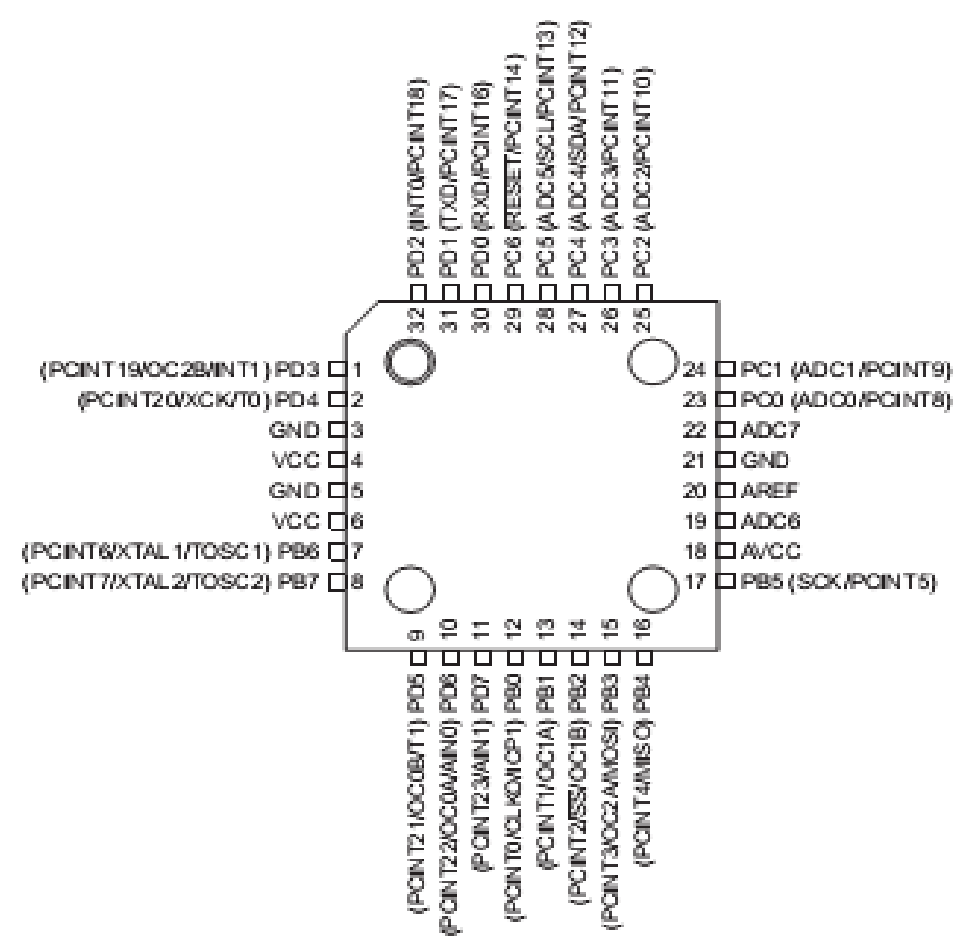

Figure 4.12 Pinout of ATMEGA328P

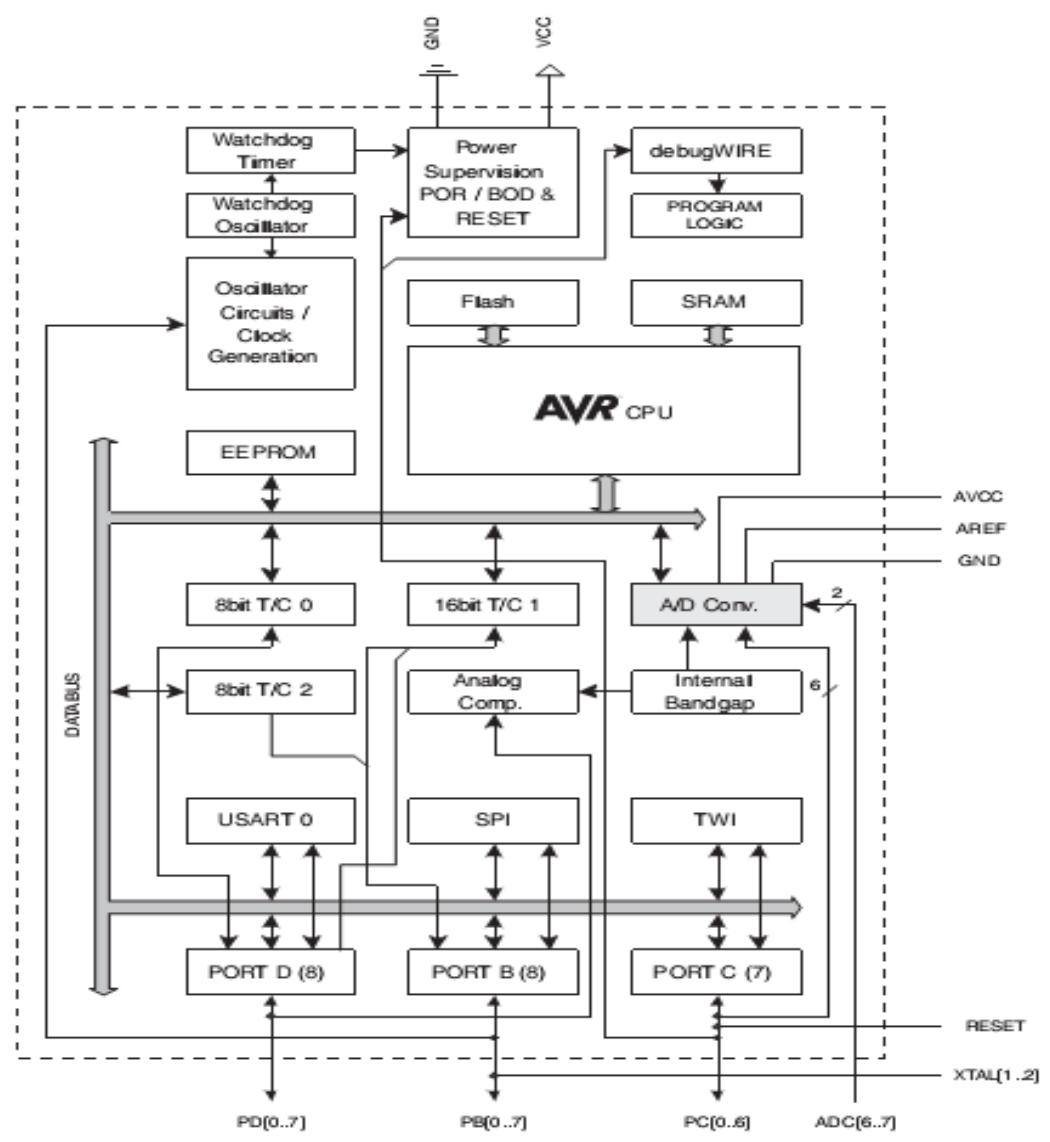

Figure 4.13 block diagram of ATMEGA328P 
- Low Power AVR 8-Bit Microcontroller

- Advanced RISC Architecture

- 131 Powerful Instructions - Most Single Clock Cycle Execution

- 32 x 8 General Purpose Working Registers

- Fully Static Operation

- Up to 20 MIPS Throughput at $20 \mathrm{MHz}$

- On-chip 2-cycle Multiplier.

- 32K Bytes of In-System Self-Programmable Flash program memory

- $1 \mathrm{~K}$ Bytes EEPROM

- $2 \mathrm{~K}$ Bytes Internal SRAM

- Two 8-bit Timer/Counters

- One 16-bit Timer/Counter

- 8-channel 10-bit ADC

- Programmable Serial USART

- Master/Slave SPI Serial Interface

- On-chip Analog Comparator

- Operating voltage : $1.8-5.5 \mathrm{~V}$

- Speed grade : 0 - $20 \mathrm{MHz} @ 1.8$ - 5.5V

\subsubsection{ITG 3205 Digital gyroscope}
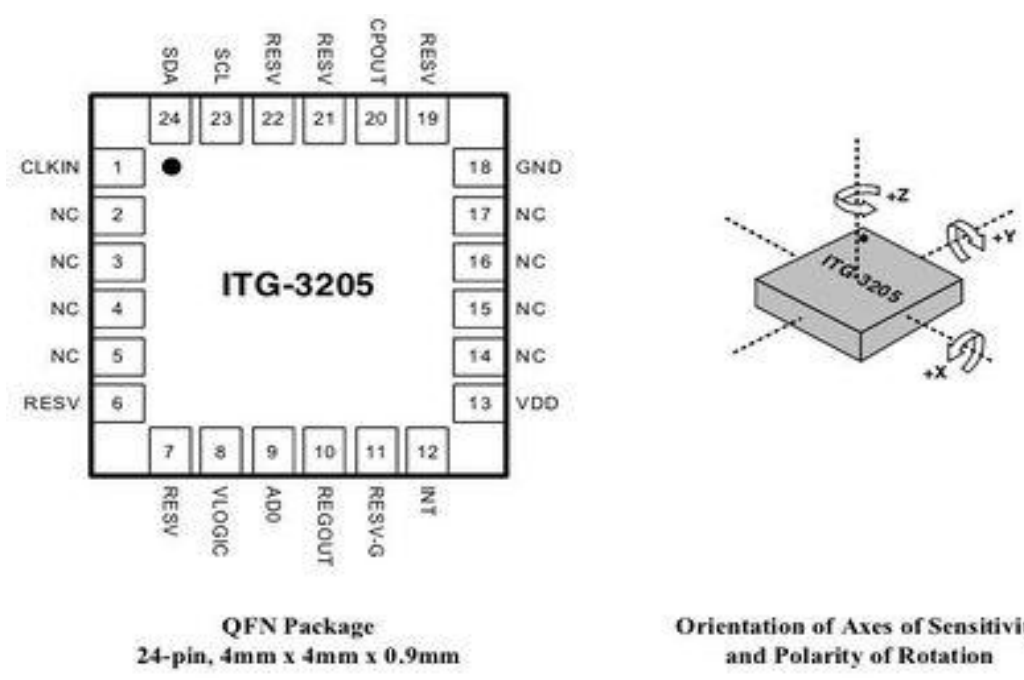

Figure 4.14 Digital Gyroscope 
ITG 3205 is a newer version of Invensense's ITG 3200 triple-axis digital output gyroscope chips.

- Three 16 bit ADC's for digitizing the gyro outputs

- Digital-output X-, Y-, and Z-Axis angular rate sensors (gyros) on one integrated circuit

- Low 6.5mA operating current consumption for long battery life

- Wide VDD supply voltage range of $2.1 \mathrm{~V}$ to $3.6 \mathrm{~V}$

- Standby current: $5 \mu \mathrm{A}$

- Fast Mode I2C (400kHz) serial interface

- Dimensions: $15.5 \times 15.5 \mathrm{~mm}$

- Range : -2000 to $2000 \mathrm{deg} / \mathrm{s}$

A gyroscope measure angular velocity, in other words the rotational speed around the three axes. The output of a gyroscope is given in radians per second or degrees per second.

The gyroscope tends to drift a lot, which means that if you start rotating the sensor, the gyroscope will output the angular velocity, but when you stop it doesn't necessarily go back to $0 \mathrm{deg} / \mathrm{s}$. If you then just used the gyroscope readings you will get an orientation that continues to move slowly (drifts) even when you stopped rotating the sensor.

So gyro should be accompanied by accelerometer. 


\subsubsection{BMA 180 Digital Triple Axis Accelerometer}

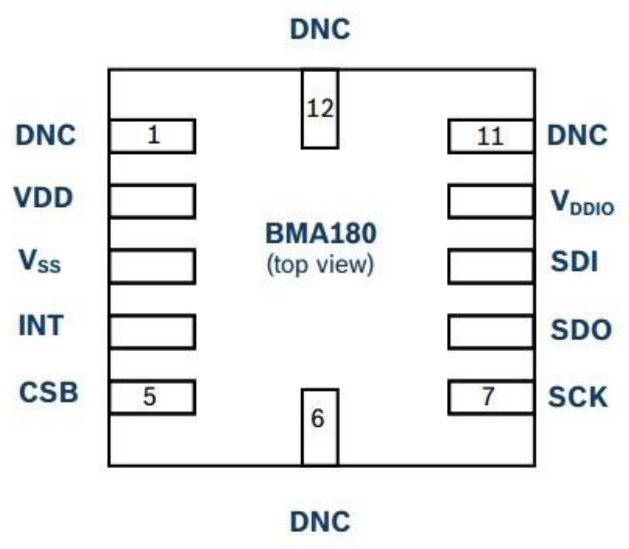

Figure 4.15 Digital accelerometer

This is a breakout board for Bosch's BMA180 three-axis, ultra-high performance digital accelerometer

- Wide variety of measurement ranges $( \pm 1 \mathrm{~g}, 1.5 \mathrm{~g}, 2 \mathrm{~g}, 3 \mathrm{~g}, 4 \mathrm{~g}, 8 \mathrm{~g}$ and $16 \mathrm{~g}$

- 14 or 12-bit ADC conversion

- 2 selectable I2C addresses

- 2 main standard modes: low-noise and low-power

- Dimensions: 0.80 x 0.40"
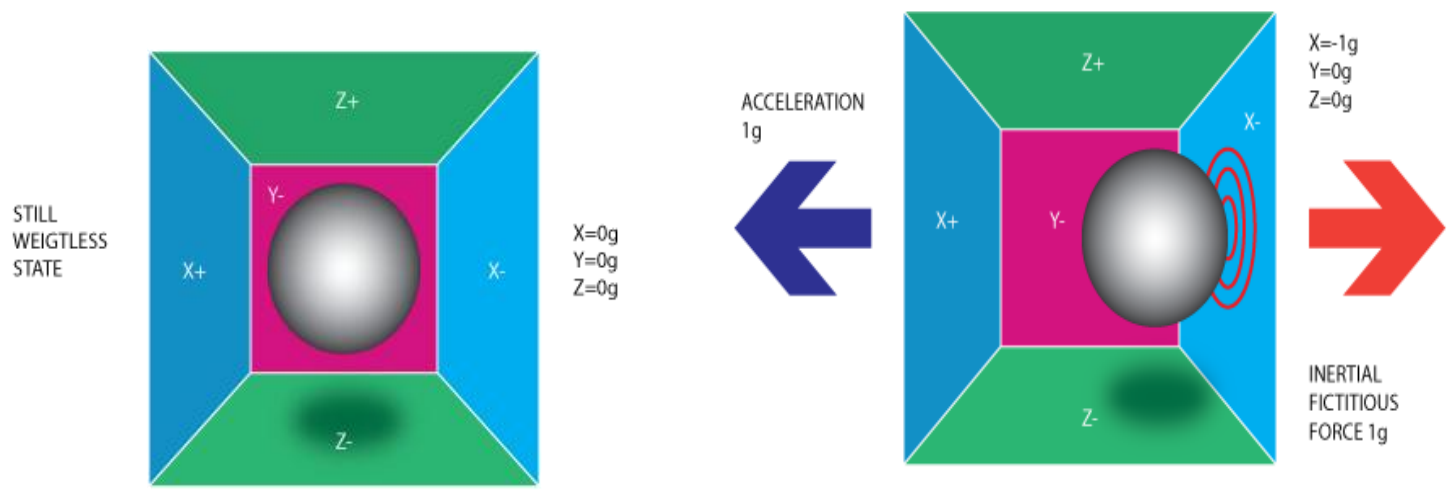

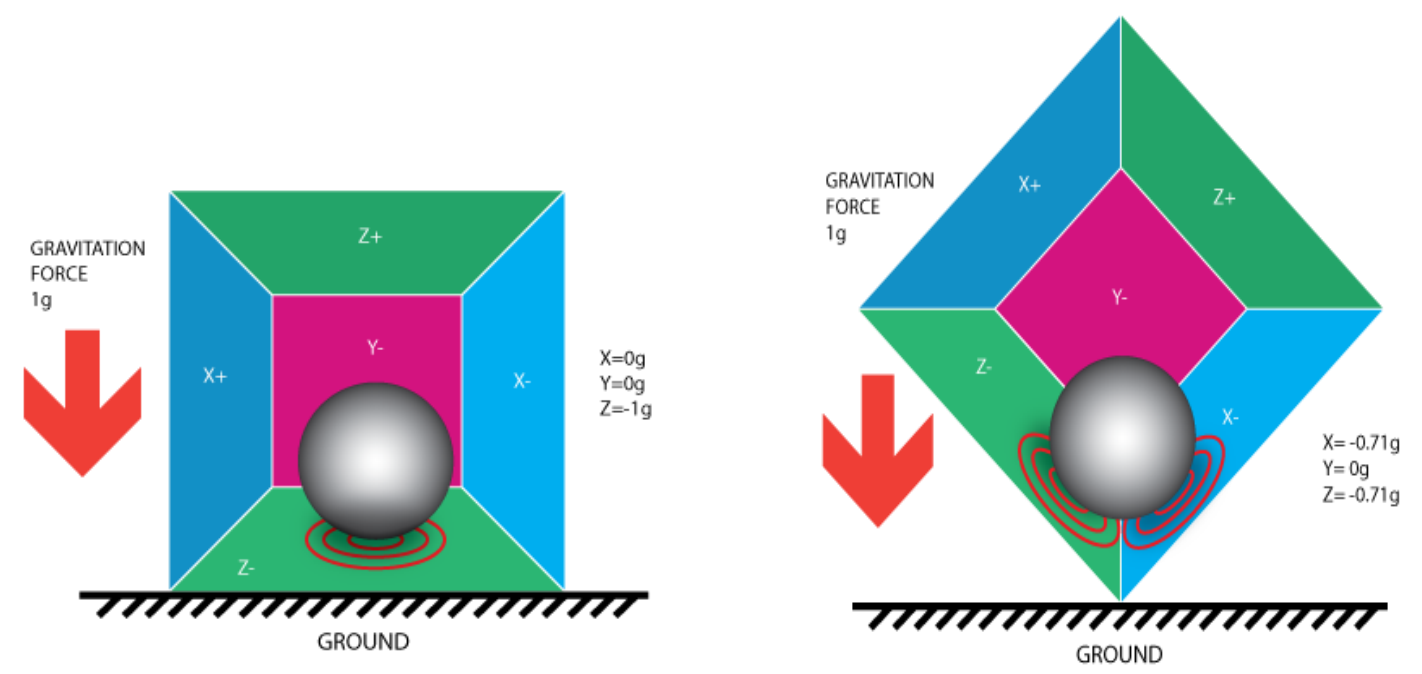

Figure 4.16 Accelerometer example

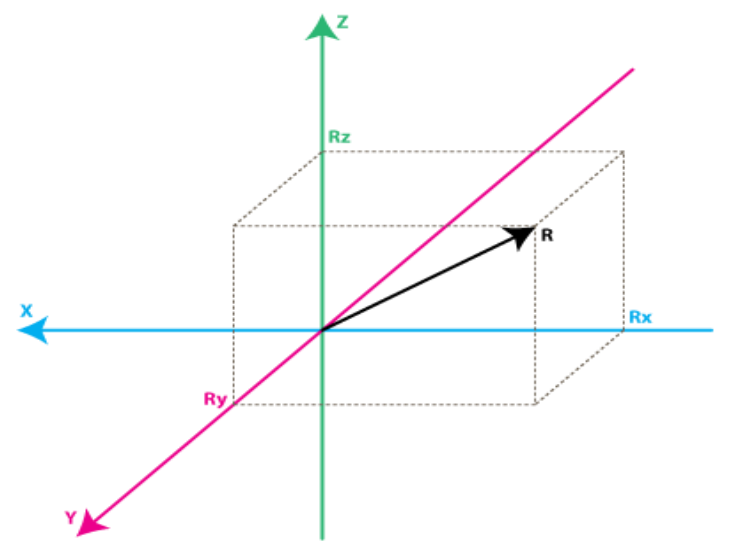

Figure 4.17 Accelerometer force vector

$$
\begin{gathered}
\mathrm{R}^{\wedge} 2=\mathrm{Rx}^{\wedge} 2+\mathrm{Ry}^{\wedge} 2+\mathrm{Rz}^{\wedge} 2 \\
\mathrm{Rx}=(\text { AdcRx } * \text { Vref } / 1023-\text { VzeroG }) / \text { Sensitivity } \\
\mathrm{Ry}=(\text { AdcRy } * \text { Vref } / 1023-\text { VzeroG }) / \text { Sensitivity } \\
\mathrm{Rz}=(\text { AdcRz } * \text { Vref } / 1023-\text { VzeroG }) / \text { Sensitivity }
\end{gathered}
$$

The accelerometers measures acceleration as the name indicates. Now you would think, why the heck do we need to measure acceleration to know the orientation? But yet again there is another law of physics the gravity. The gravity is actually a downwards acceleration towards the center of earth, which to all objects makes a downward force keeping the objects on the surface. The accelerometer is actually measuring force, so the downwards gravity acceleration will also be measured by the accelerometer. 
As the accelerometer sensor can measure the acceleration in three directions we can actually calculate how the accelerometer is oriented against the surface.

The accelerometer, then enough to measure the orientation? The problem lies in the way the accelerometer works, because it isn't very stable. If only the accelerometer were used to calculate the orientation even the smallest movements of the accelerometer will mess up the orientation. So if mounted on a Quadcopter with vibrating motors it will be no good.

So accelerometer is used in parallel with gyro.

\subsubsection{HMC5883L Magneto Resistive Sensor}

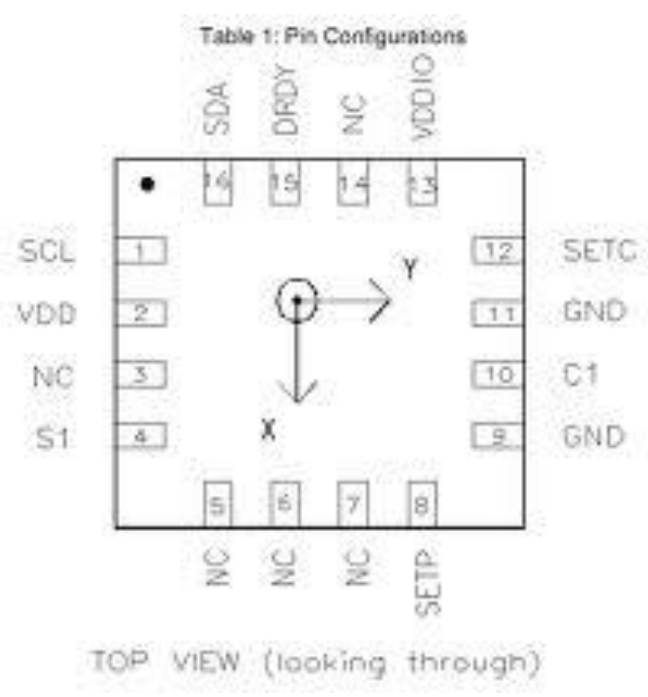

Figure 4.18 magneto resistive sensor

- 3 Axis Magneto resistive Sensors.

- 12 Bit ADC

- Low Voltage Operations (2.16 to 3.6V)

- Low Power Consumption $(100 \mu \mathrm{A})$

- I2C Digital Interface.

For the Yaw rotational movement the accelerometer can't be used as the reference sensor as it could with the Roll and Pitch movement. Instead a magnetometer is sometimes used. A 3-axis magnetometer measures the magnetic field that affects the sensor in all three directions. As the earth contains a magnetic field on the North- and South Pole, the magnetic sensor can be used to determine where north and south is located. The locations of these poles can then be used as a reference 
together with the angular velocity around Yaw from the gyroscope, to calculate a stable Yaw angle.

\subsubsection{FTDI Basic Programmer}

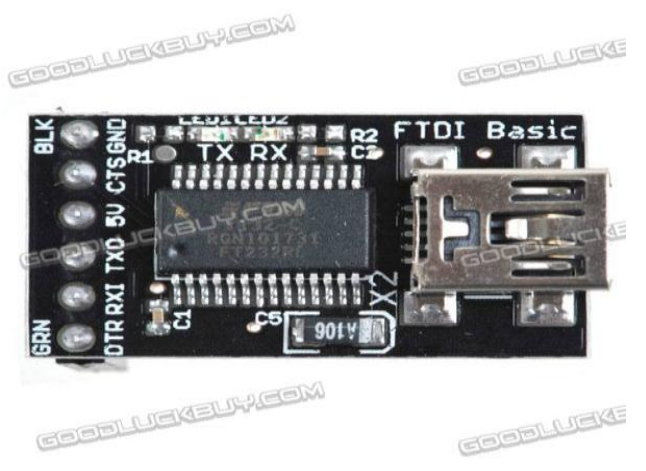

Figure 4.19 FTDI BASIC PROGRAMMER

This is a basic breakout board for the FTDI FT232RL USB to serial IC. The pinout of this board matches the FTDI cable to work with official Arduino and cloned 5V Arduino boards. It can also be used for general serial applications. The major difference with this board is that it brings out the DTR pin as opposed to the RTS pin of the FTDI cable. The DTR pin allows an Arduino target to auto-reset when a new Sketch is downloaded. This is a really nice feature to have and allows a sketch to be downloaded without having to hit the reset button. This board will auto reset any Arduino board that has the reset pin brought out to a 6-pin connector.

The pins labeled BLK and GRN correspond to the colored wires on the FTDI cable. The black wire on the FTDI cable is GND, green is CTS. Use these BLK and GRN pins to align the FTDI basic board with your Arduino target.

This board has TX and RX LEDs that make it a bit better to use over the FTDI cable. You can actually see serial traffic on the LEDs to verify if the board is working.

One of the nice features of this board is a jumper on the back of the board that allows the board to be configured to either $3.3 \mathrm{~V}$ or $5 \mathrm{~V}$ (both power output and IO level). This board ship default to $5 \mathrm{~V}$, but you can cut the default trace and add a solder jumper if you need to switch to $3.3 \mathrm{~V}$. 


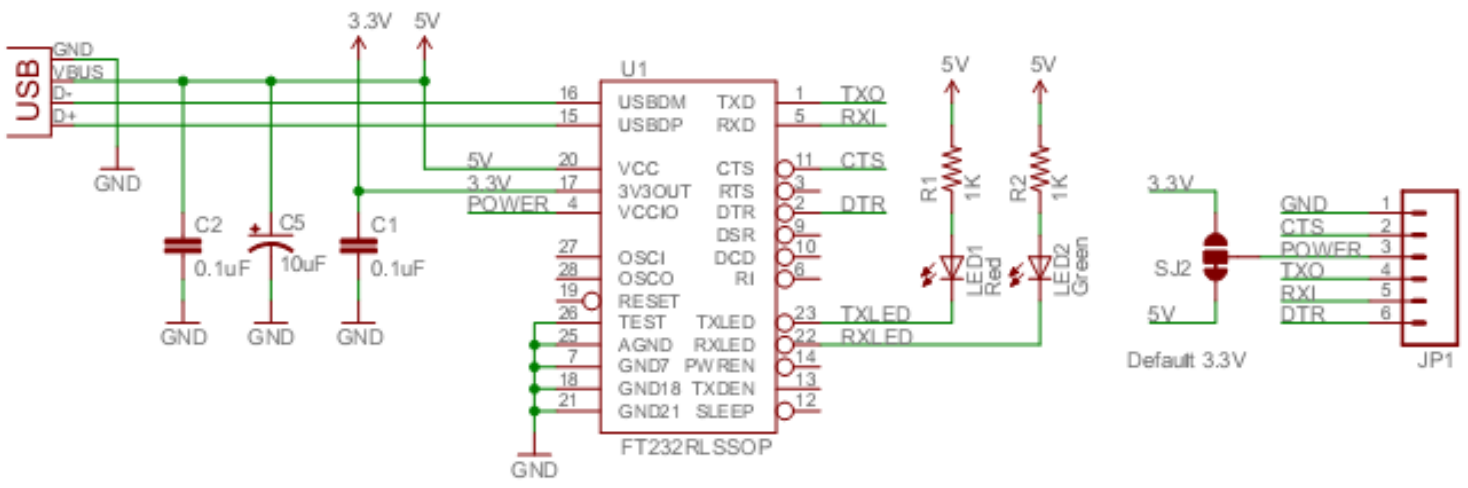

Figure 4.20 schematic of FTDI

\subsection{0 calculations basing on selected components}

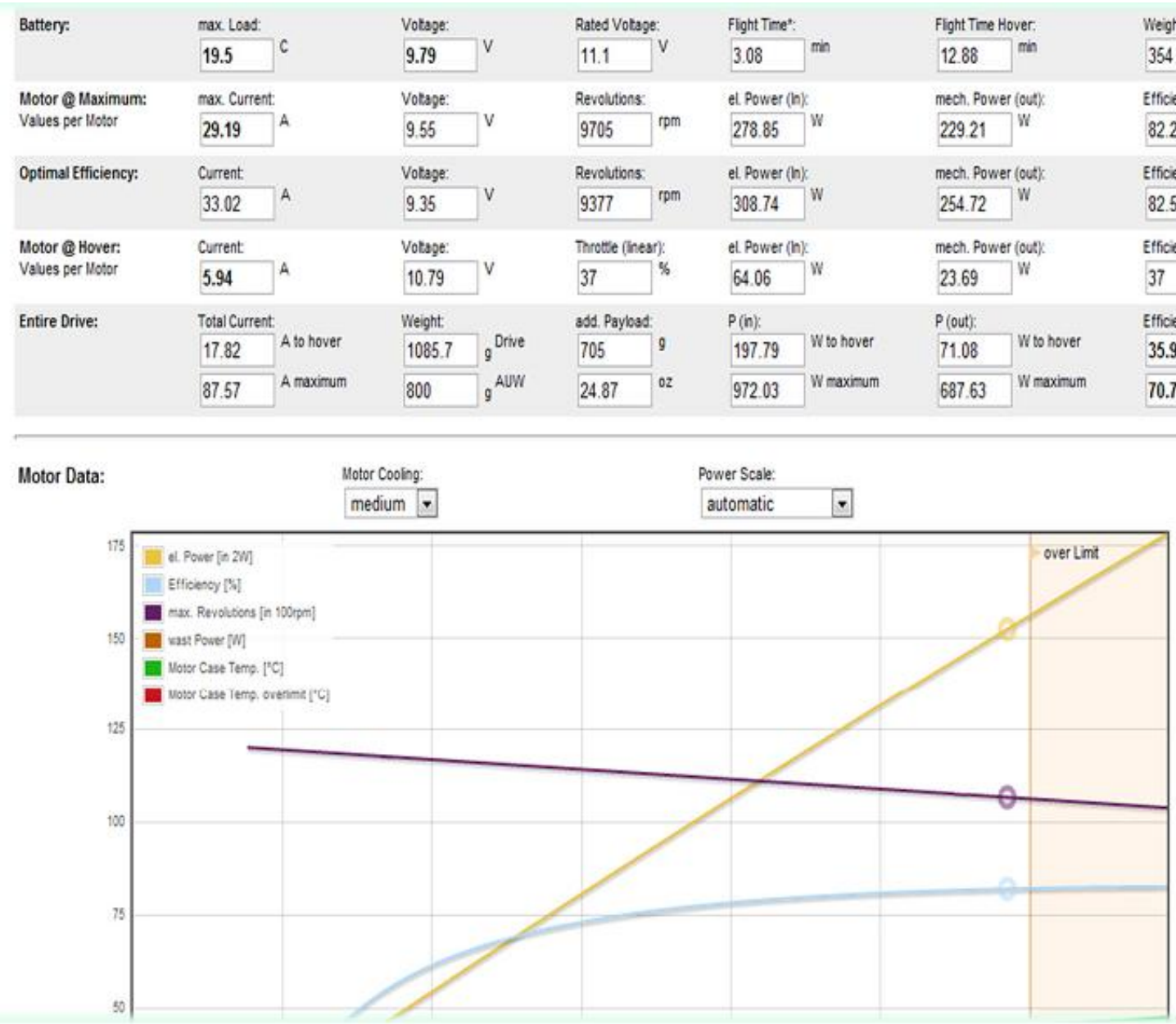

Figure 4.21 Calculations

Source: http://www.ecalc.ch/xcoptercalc.htm?ecalc\&lang=en 
'To accomplish great things, we must not only act, but also dream, not only plan, but also believe.'

-Anatole France

\section{CHAPTER 5}

\section{SOFTWARES USED}

\subsection{ARDUINO}

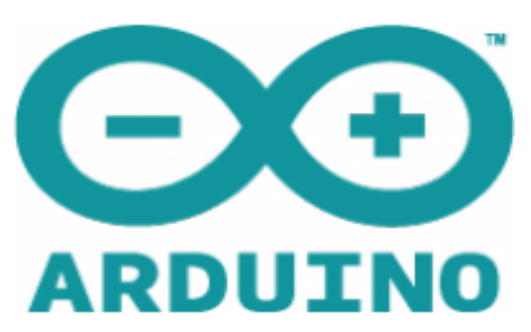

Figure 5.1 Arduino logo

The open-source Arduino environment makes it easy to write code and upload it to the I/O board. It runs on Windows, Mac OS X, and Linux. The environment is written in Java and based on Processing, avr-gcc, and other open source software.

We can download the Arduino environment from the internet with url www.arduino.cc 


\subsubsection{How to use Arduino}

1. Launch the Arduino application

Double-click the Arduino application. (Note: if the Arduino software loads in the wrong language, you can change it in the preferences dialog.)

2. Open the program needed to upload to microcontroller.

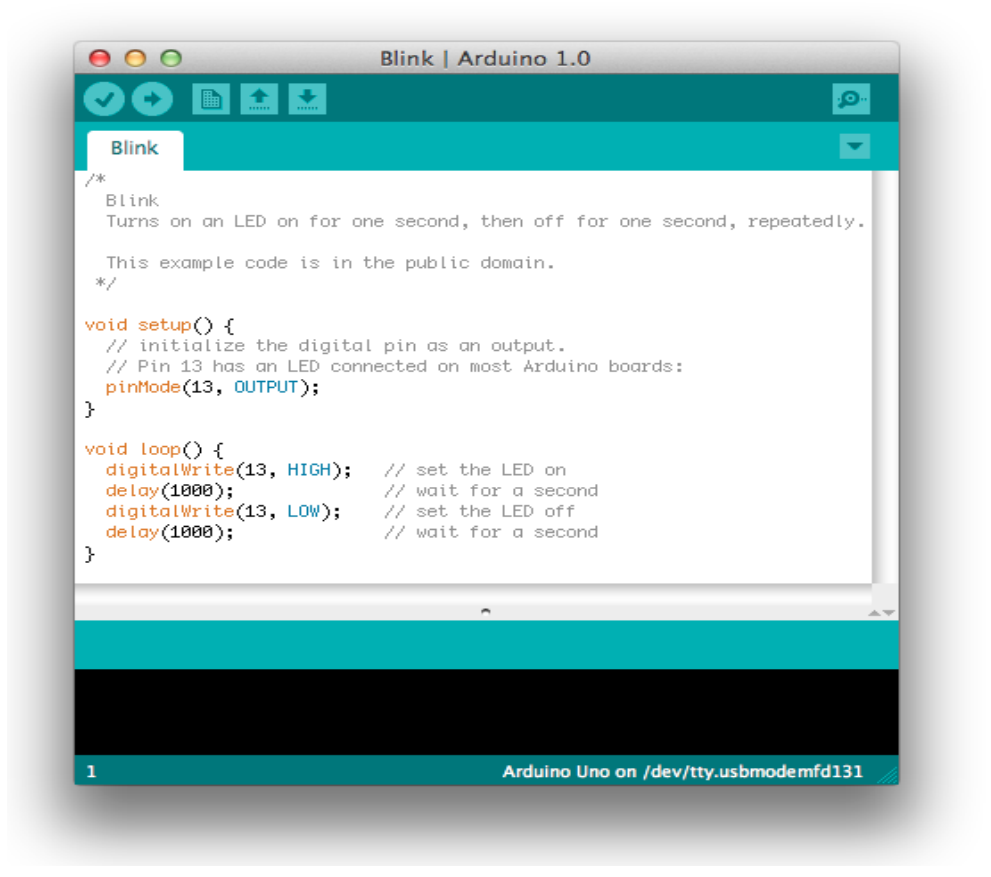

Figure 5.2 Arduino environment

3. Select your board

You'll need to select the entry in the Tools > Board menu that corresponds to your Arduino. 


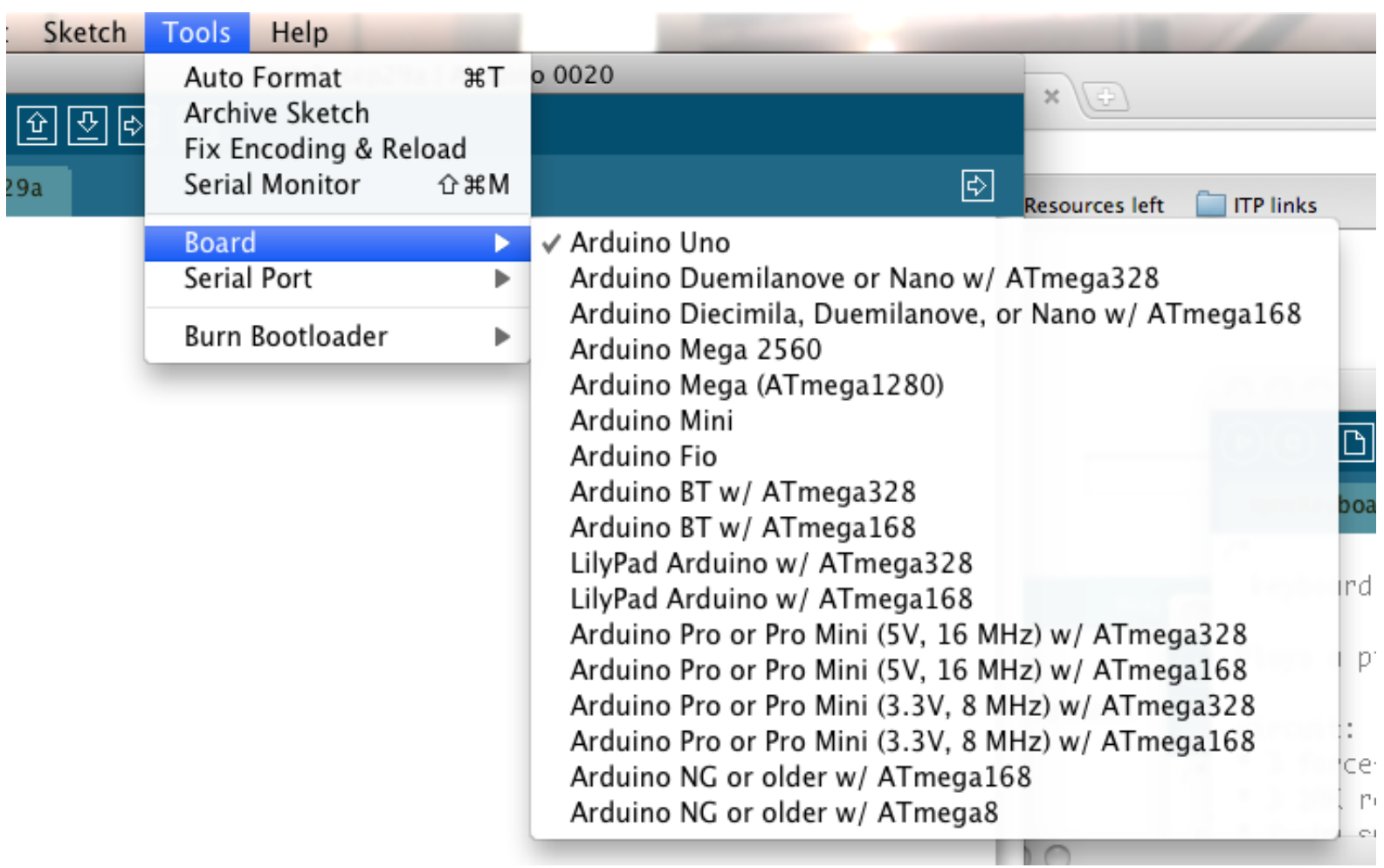

Figure 5.3 Selecting an Arduino Uno/microcontroller

\section{Select your serial port}

Select the serial device of the Arduino board from the Tools | Serial Port menu. This is likely to be COM3 or higher (COM1 and COM2 are usually reserved for hardware serial ports). To find out, you can disconnect your Arduino board and reopen the menu; the entry that disappears should be the Arduino board. Reconnect the board and select that serial port.

5. Upload the program

Now, simply click the "Upload" button in the environment. Wait a few seconds - you should see the RX and TX leds on the board flashing. If the upload is successful, the message "Done uploading." will appear in the status bar.

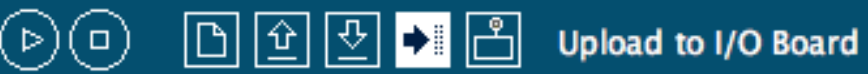




\subsection{MULTIWII CONFIG 2_1}

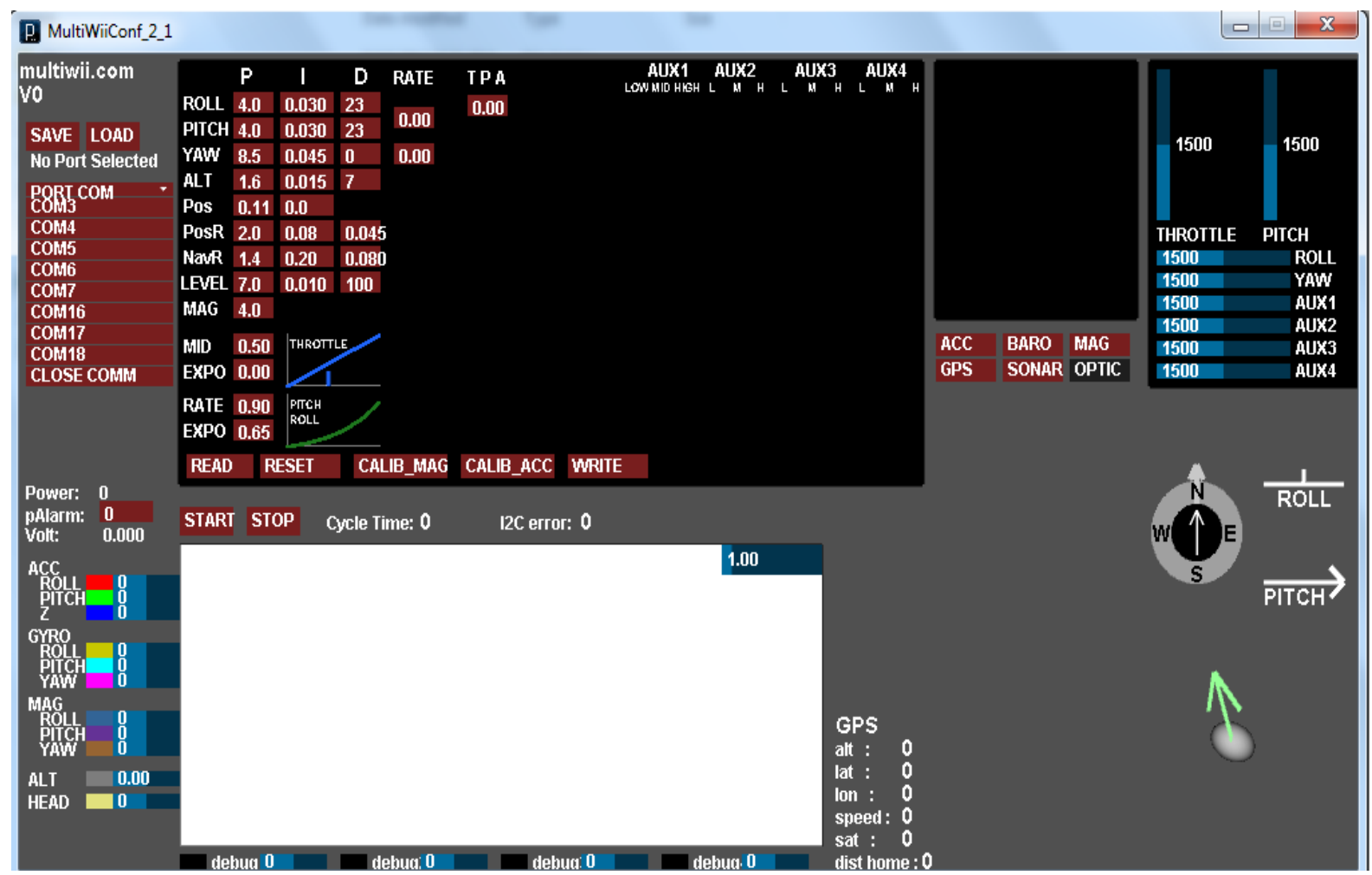

Figure 5.4 multiwii configuration screen

Multiwii code comes with a GUI (Graphical User Interface) which helps the user to setup:

- Sensor recognition

- Accelerometer/gyro calibration

- PIDs

- Rx channels

\subsubsection{Parameter explanation}

\subsubsection{RC rate and $\mathrm{RC}$ expo}

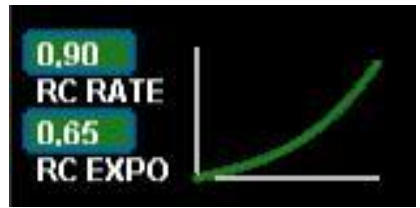


$\underline{\mathrm{RC} \text { rate: }}$ defines the sensibility of the multicopter for PITCH and ROLL RC stick. If you feel your multi too reactive, decrease it. If you want to improve its responsiveness, increase this parameter.

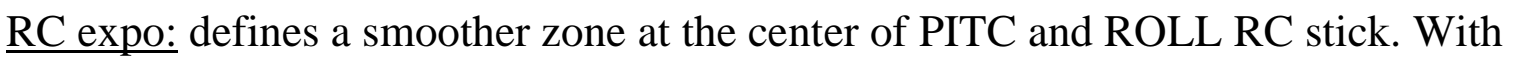
this function, it's possible to have both a good precision and good amplitude.

$0=$ no expo

$1=$ full expo

If you don't understand really those parameters, I encourage you to read your TX documentation, as those functions are also generally supported by the TX. It's better to support them directly in multiwii because the resolution is better this way.

\subsubsection{2 $P$ and I Level}

\section{LEVEL 14,0$] 0,045$}

Defines the acc influence in stable mode only (this parameter has no effect if the stable mode is disable)

If the multi is not stable once activated, reduce $\mathrm{P}$.

\subsubsection{PITCH/ROLL/YAW PID and rate}

\begin{tabular}{l|l|l|l|l|}
\hline & P & I & D & RATE \\
ROLL & 4,0 & 0,030 & 15 & 0,00 \\
PITCH & 4,0 & 0,030 & 15 & 0,0 \\
YAW & 8,0 & 0,000 & 0 & 0,00 \\
\hline
\end{tabular}

For a very stable multicopter with a solid attitude, the PID settings must be set high. But if you want to do some acrobatics with these settings, the multi starts to wobble in fast translation or when you decide to shake it too much. One solution is to decrease the PID, but it is to the detriment of static stability.

So instead of implementing a switch between 2 PID settings, there are now 2 options in the GUI to define the way we should decrease PID, depending on ROLL/PITCH/YAW stick deviation

The two boxes on the right define the rate of cancellation of the nominal PID (the one which is used on neutral ROLL/PITCH/YAW stick position) in relation with 
ROLL/STICK/YAW deviation. In fact only $\mathrm{P}$ and D parameters are impacted in the transformation. The purpose of this setting is not really to gain more stability, but to gain more maneuverability. $0=$ soft rate (for FPV or beginners); $0.4=$ soft acro $; 0.7=$ fast acro ; 1 = insane rate. If you are not familiar with this, just keep the default values (0).

\subsubsection{THROTTLE rate}

\section{Throttle PID}

attenuation

0,00

This box defines the rate of cancellation of the nominal PID (the one which is used on neutral ROLL/PITCH stick position) in relation with Throttle stick. The purpose of this curve is to gain more stability when you are in a situation to use more throttle than needed to just maintain a lift. This is typically the case in fast translation.

If you are not familiar with this, just keep the default values (0)

\subsubsection{How to activate options

AUX1 AUX2
LOWID HIEH LOW MID HEH
LEVEL
BARO
MAG
CAMSTAB
CAMTRK

Depending on your configuration, you can easily activate/deactivate options via 2 switches on your RC TX.

The 2 switches correspond to channel 5 (AUX1) and channel 6 (AUX2). LEVEL is the auto level feature (require an ACC)

BARO allows to keep a constant altitude once activated (require a barometer) MAG allows to keep a perfect heading direction once activated (require a magnetometer)

CAMSTAB: if activated, the PITCH\&ROLL servo output will follow the inclination of the multi (require an ACC + the code activation via \#define statement)

CAMSTRIG: if activated, a servo output will trigger repetitively a sequence where we can define the duration on the HIGH/LOW position. 
To activate a function, you must check the white box at the intersection of the required option (row) and at the switch state you want to enable (column).

It's possible to make a complex mix.

If you want to force an option permanently, it's possible to check all the white boxes, even without a RC channel connected.

(=> This way, you don't need a 5 or 6 channels $\mathrm{RC}$ receiver).

\subsubsection{Sensor detection/usage}

NUNCHUK

ACC

BARO

ACTINE

OFF

MAG

OFF

On this screen, the left column resumes the detection of sensors.

The right column precise if the sensor is currently use or not (depending on the checked options) 
'Sometimes things become possible if we want those bad enough'

-T.S.Eliot

\section{CHAPTER 6}

\section{ELECTRICAL CONNECTIONS}

\subsection{Introduction}

When we have all the components required to build Quadcopter then the next step to move forward is to connect all the components according to their specifications, pin outs. This chapter is divided into two phases.

In the first phase the schematic diagram of the overall connections is given and in latter phase each component connection with the other is given in detail.

\subsection{Schematic diagram:}




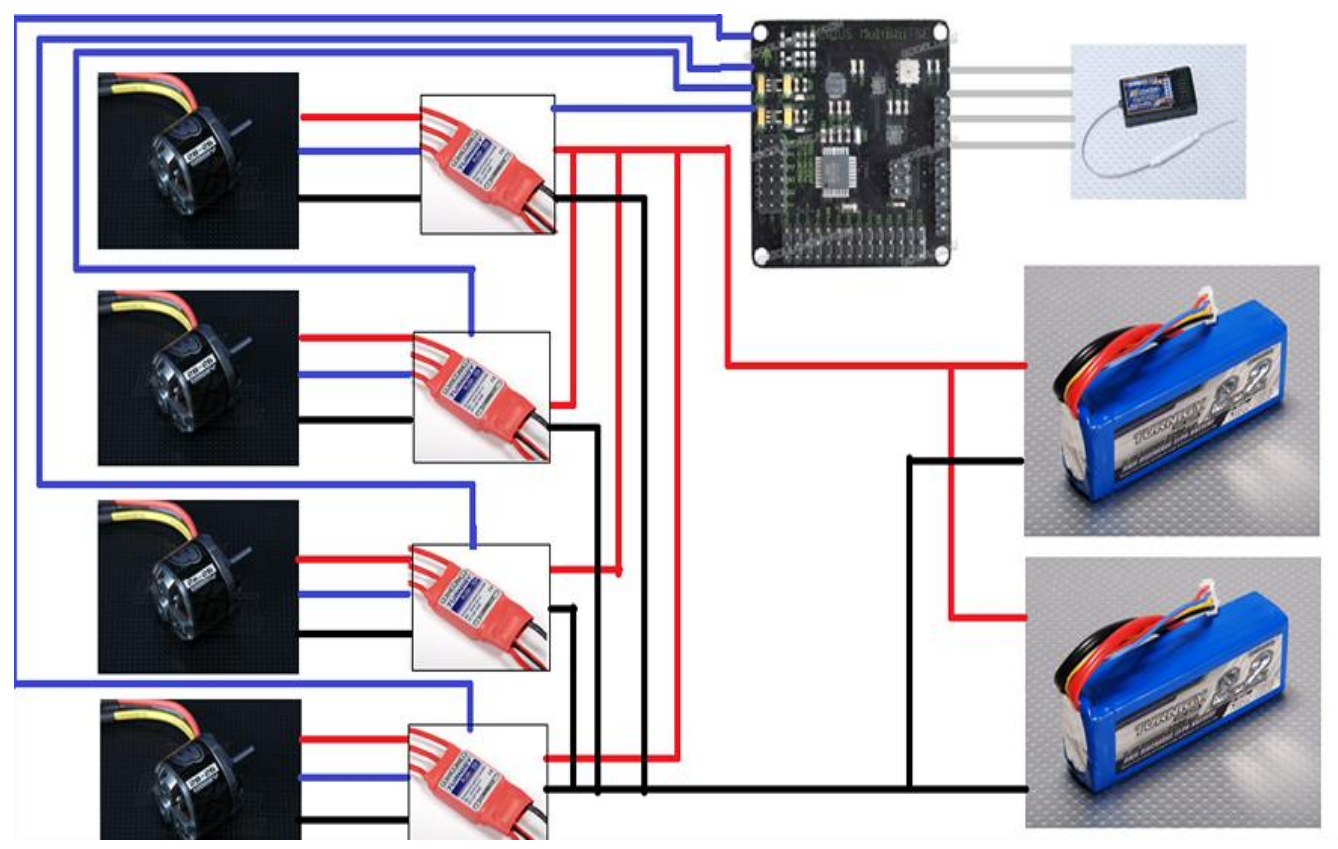

Figure 6.1 Schematic diagram

\subsection{Building Quadcopter}

\subsubsection{The Frame}

Every part in a Quadcopter design works together and the frame is the one joining all of them. The frame can be designed in many ways with many different kinds of materials. The important things are to make it rigid and to minimize the vibrations coming from the motors.

A Quadcopter frame consists of two to three parts which don't necessarily have to be of the same material:

- $\quad$ The center part where the electronics and sensors are mounted

- $\quad$ Four arms mounted to the center part

- $\quad$ Four motor brackets connecting the motors to the arms

There are three kinds of materials that I recommend using for a Quadcopter frame:

- $\quad$ Carbon Fiber

- Aluminium

- $\quad$ Plywood or MDF 
Carbon fiber is the most rigid and vibration absorbent of the three materials but is also by far the most expensive.

Most of the times the arms used in Quadcopter are made of hollow aluminium square rails which make the Quadcopter relatively light weight but still make it rigid. The problem with these hollow aluminum rails are the vibrations, as they aren't damped and will therefore vibrate to the center part and maybe mess up the sensor readings.

Instead solid MDF plates could be cut out for the arms as they will absorb the vibrations much better than the aluminium. Unfortunately we have another problem then, as the MDF plates are not very rigid and will break if the Quadcopter falls to the ground.

As for the center part everything from carbon fiber, aluminium or plywood can be used. We have used fiber type material which is very rigid and light in weight.

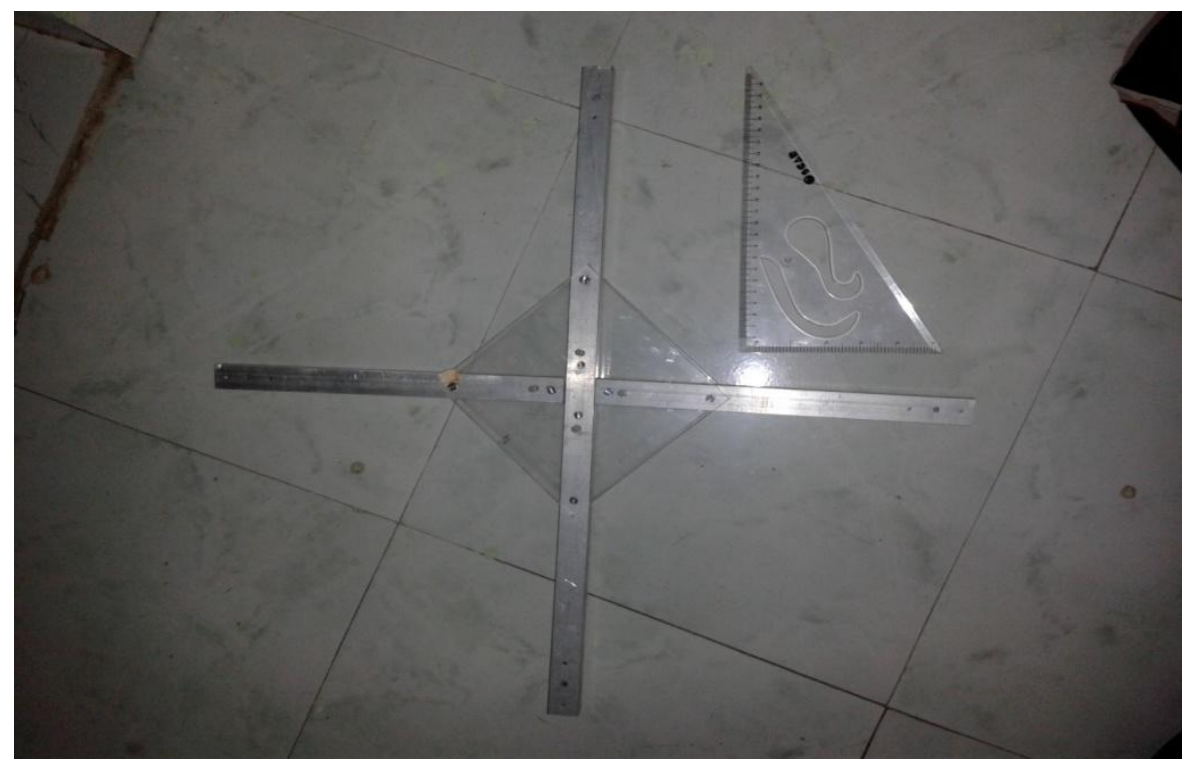

Figure 6.2 Quadcopter frame

Our frame has a tip-to-tip length of around $55 \mathrm{cms}$. 


\subsubsection{Mounting the motors}

After constructing the frame the next job is to mount the motors on to the frame. Here we need to take lot of care as when motors rotate with very high speeds, they cause vibrations and those vibrations may cause unwanted deflections in the sensors used. So in order to damp the vibrations from the motors we need to use some rubber damping material between motors and the frame.

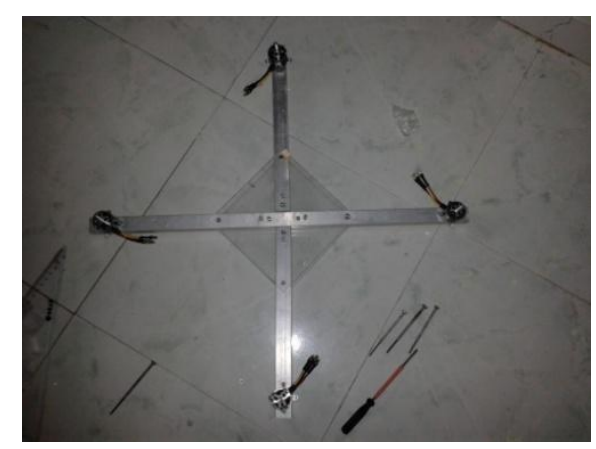

Figure 6.3 Motors mounted onto the frame

\subsubsection{Choosing proper propellers}

We have two types of propellers, one with right tilting and the other with left tilting. In our Quadcopter we have two motors rotating in clockwise and other pair rotating in anticlockwise rotations.

So, here the propellers with right tilting are fixed to pair of motors rotating in clockwise direction and the propellers with left tilting are fixed to pair of motors rotating in anti-clockwise direction.

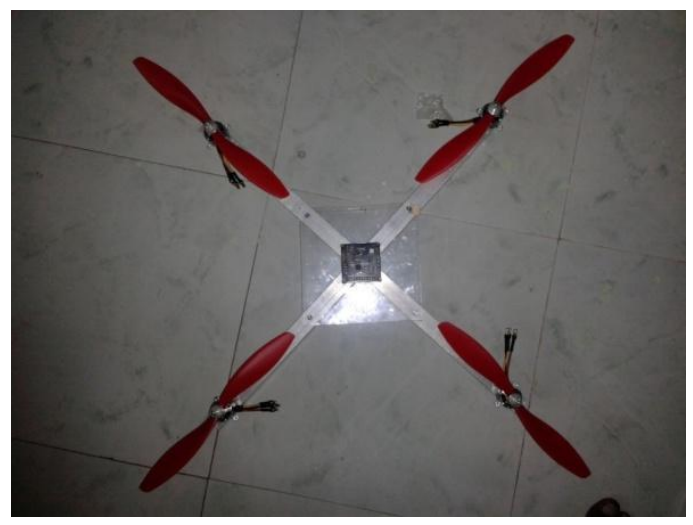

Figure 6.4 Proper tilted Propellers connected to proper motors

\subsubsection{ESC connection to motors}

We have electronic speed controllers (ESCs) to control the speed of the motors. The direction of rotation of motors is based upon the connection of ESC to motor. 
Brushless DC motors will have three terminals and ESC has its output as 3 terminals so these three terminals are connected with motor three terminals. To change the direction of rotor we need to change any two of these three output terminals.

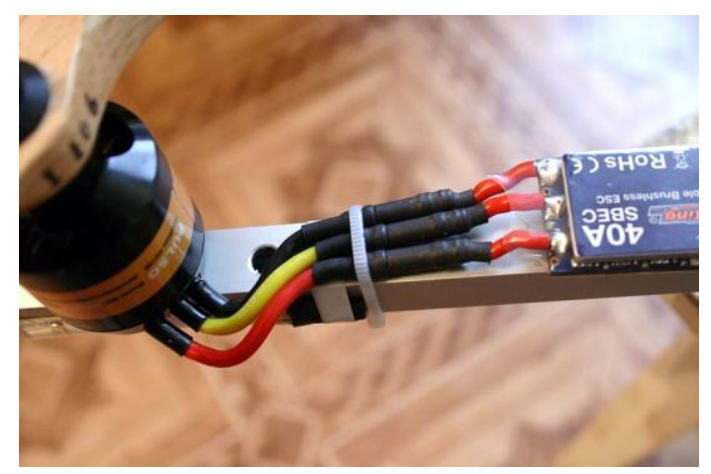

Figure 6.5 ESC to motor wiring

Battery is connected to motor via ESC. So one input to ESC will be given from battery. The other control input to ESC will get from the flight control board.

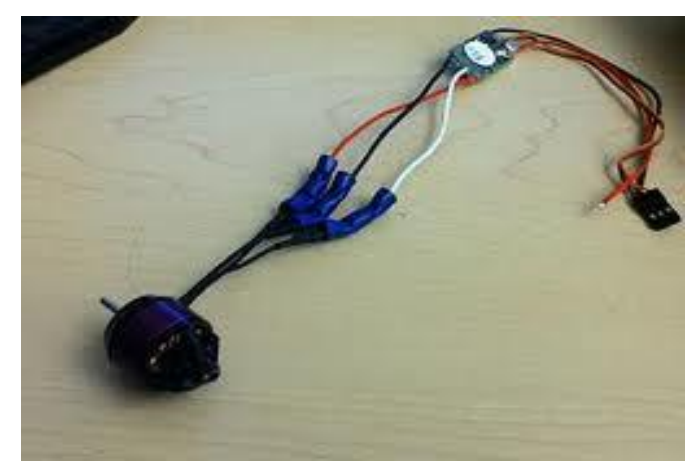

Figure 6.6 ESC to motor

Here, we have four motors and therefore four ESCs are needed to be connected to single battery so we need to think of a wire connection to get a single battery connected to four ESCs.

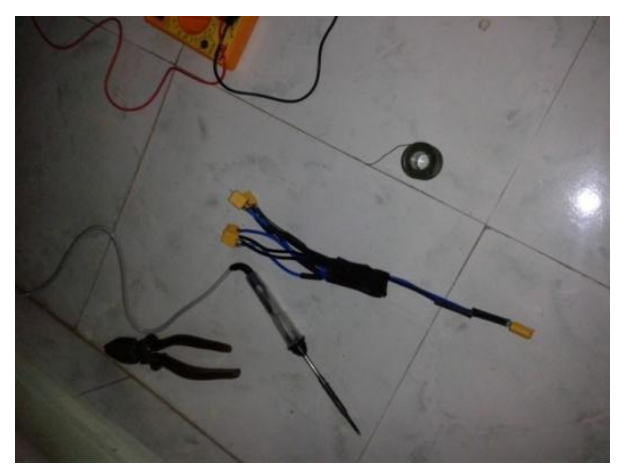

Figure 6.7 Wiring to connect battery to four ESCs 


\subsubsection{Throttle calibration}

In order to calibrate the throttle we need to connect the ESC to receiver $\left(3^{\text {rd }}\right.$ pin -Throttle).

- Connect the ESC output to the motor.

- Turn on the Transmitter with the throttle in MAX position.

- Connect the control input of ESC to $3^{\text {rd }}$ pin of receiver.

- Connect the battery terminals of ESC to the battery.

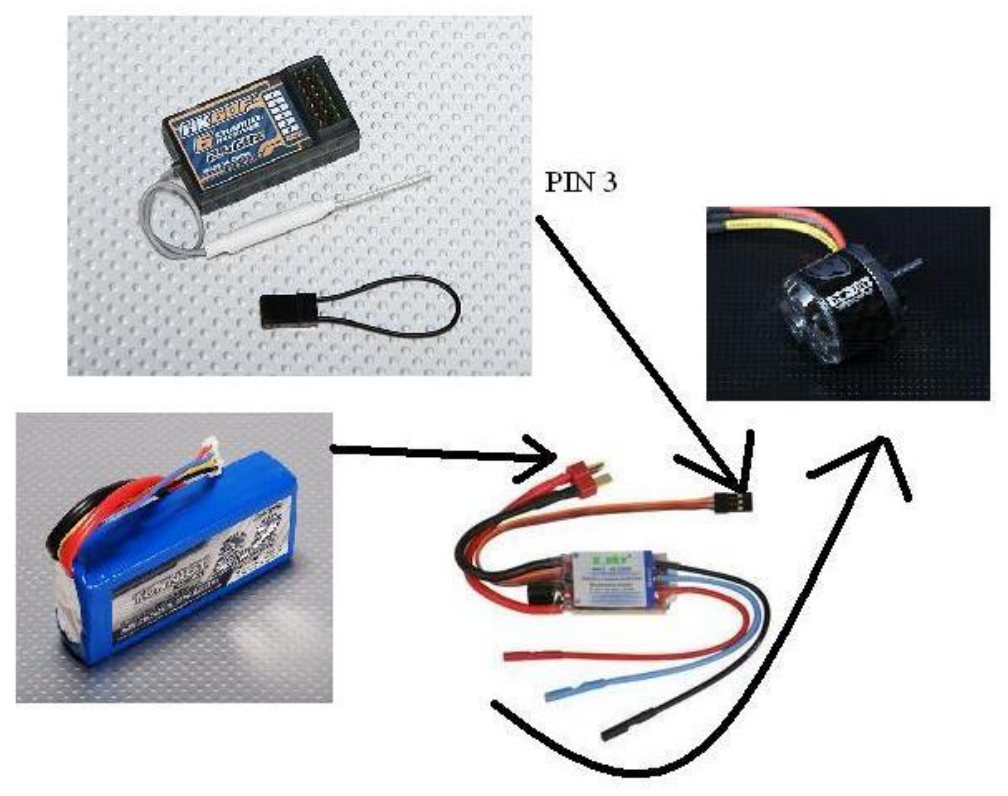

Figure 6.8 Wiring for calibration

- As we connect battery terminals to ESC we would get two beeps.

- As soon as we hear two beep sounds we should decrease the throttle to MIN position.

- After this again we hear two beeps, this indicates us that the ESC calibration has been completed.

- After this it emits two sets of audible tones in succession indicating the status of its programming state.

- The first set of tones denotes the number of cells in the LiPo pack connected to the ESC. (Three beeps indicate a 3 cell LiPo pack while 4 beeps indicate a 4 cell LiPo pack).

- The second set denoting Brake status (one beep for Brake "ON" and two beeps for Brake "OFF").

- The ESC is now ready for use. 


\subsubsection{Connecting receiver to flight control board}

The receiver has 6 channels out of which we use 5 channels to connect the receiver and flight control board. For each connection there are three terminals (VCC, GND, SGNL). The flight control board should be mounted on the Quad copter so that the arrow direction marked 'front' on board should be directed to front of Quadcopter.

Receiver

Pin 1 Aileron

Pin 2 Elevator

Pin 3 Throttle

Pin 4 Rudder

Pin 5 Gear digital
Flight control board

Roll

Pitch

THR

Yaw

Aux pin

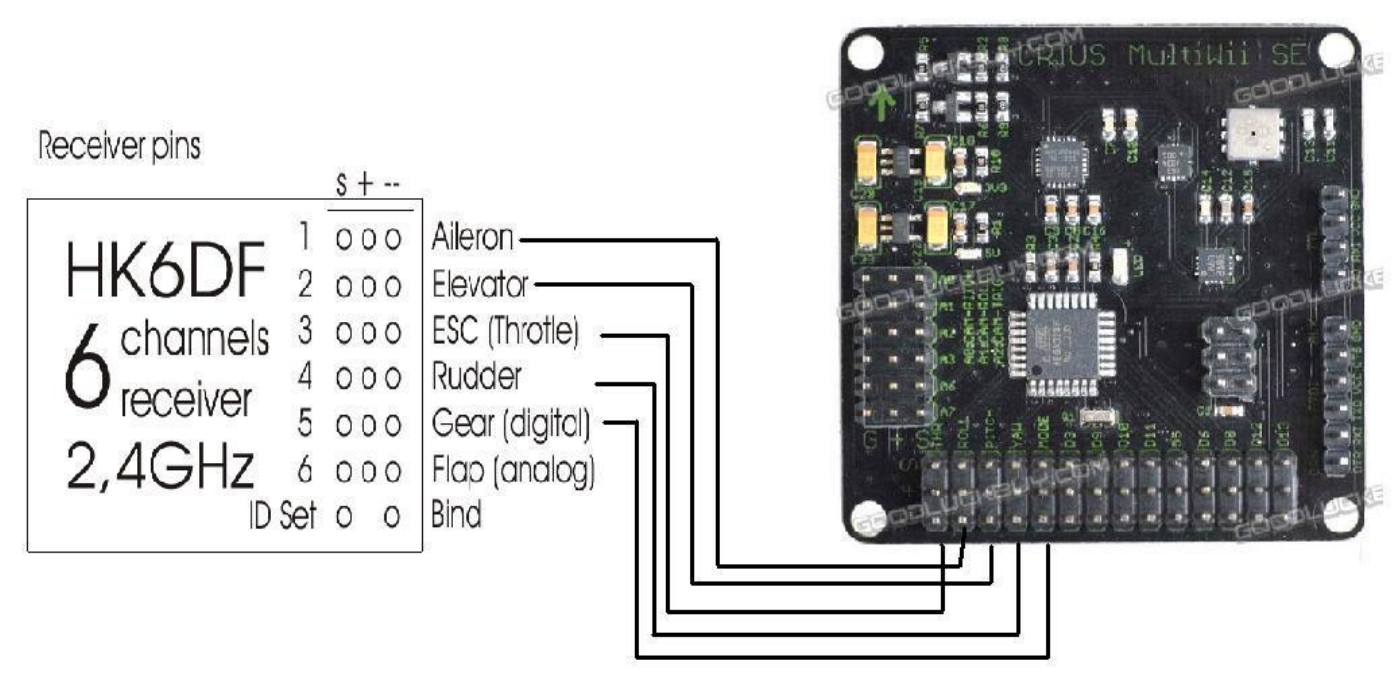

Figure 6.9 receiver and flight control board connection 


\subsubsection{Connecting motors to flight control board}

Motors are connected to flight control board via ESC. Each ESC has three terminals to be connected to flight control board (VCC, GND, and SGNL).

\section{Motor}

Top left motor (3)

Bottom Right motor (9)

Top right motor (10)

Bottom right motor (11)

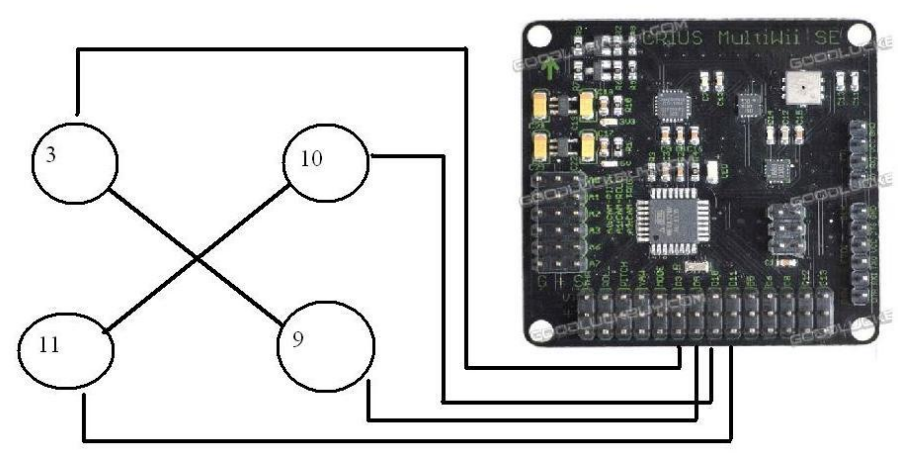

Figure 6.10 motors and flight control board connection
Flight control board

D3 pin

D9pin

D10 pin

D11 pin 
'The world is the great gymnasium where we come to make ourselves strong'

-Swami Vivekananda

\section{CHAPTER 7}

\section{PROGRAMMING AND CONFIGURATION}

\subsection{Introduction}

In this chapter we have two parts, the first part deals with the interfacing of flight control board to a computer and uploading code into the microcontroller and the second part deals with the configuration of Quadcopter.

\subsection{Programming}

\subsubsection{Connecting flight control board to computer}

The flight control board is connected to the computer with the help of FTDI basic programmer. We have 6pins on flight control board to connect the board to computer. They are DTR, RXI, TX0, VCC.CTS, and GND.

We should install the driver of FTDI programmer in the system to get the device connected to the system.

The drivers of FTDI basic programmer are available online at http://www.ftdichip.com/FTDrivers.htm 


\subsubsection{Code (MULTIWII) and uploading}

We have used open source code to upload into the microcontroller. The code we have used is MILTIWII.

MultiWii is general purpose software to control a multirotor RC model. We have used ARDUINO platform to compile and upload the code into the microcontroller.

We used 2.1 which was released in July 2012.

We can find this code at: https://code.google.com/p/multiwii/downloads/list

\subsubsection{Configuring code}

Multiwii has config.h file which is used to configure the open source code according tour specifications.

In the config.h we have updated the code according to our requirements.

The changes we have done include:

The type of multicopter: uncomment \#define QUADX

Motor min throttle: \#define MINTHROTTLE 1000

Motor Max throttle: \#define MAXTHROTTLE 1850

Min Command: \#define MINCOMMAND 900

Boards and sensor definitions: \#define CRIUS_SE

Independent Sensors

Gyro: \#define ITG3200

Accelerometer: \#define BMA180

Magnetometer: \#define HMC5883 


\subsubsection{Failsafe configuration}

In the code we have an option of failsafe feature; this option is very useful when the quadrotor loses the signal from the transmitter.

The failsafe routine of multiwii determines what is happening if the connection between RX and TX is lost.

If a loss of TX Signal is detected the copter enters stable mode after a guard time if an ACC is present and applies a configured throttle for a given time before it shuts down the motors.

There are different options that control the behavior of the multirotor after loss of TX signal:

\section{\#define FAILSAFE}

Failsafe function activated if TX signal loss found.

\section{\#define FAILSAFE_PIN}

On which pin should the FC check for a signal loss

\section{\#define FAILSAVE_DELAY}

How long does the copter need to notice a loss of TX signal?

\section{\#define FAILSAVE_OFF_DELAY}

How long does the copter try to land before switching off the motors? If you set this value to 0 the copter will shut down immediately - sometimes the safest solution. Be aware, that the copter may drift and crash into anything while trying to land.

\section{\#define FAILSAVE_THROTTLE}


How much throttle is applied while trying to land? This value is dependent on the weight of your copter, the strength of your motors and on the size of your propellers. You need to test, which value is right to enter a descending movement.

\subsubsection{Uploading code:}

After configuring the code, the code should be uploaded into the microcontroller using Arduino platform.

Before uploading we need to set some settings in Arduino platform

Tools $>$ Board-select Arduino w/ Atmega328P

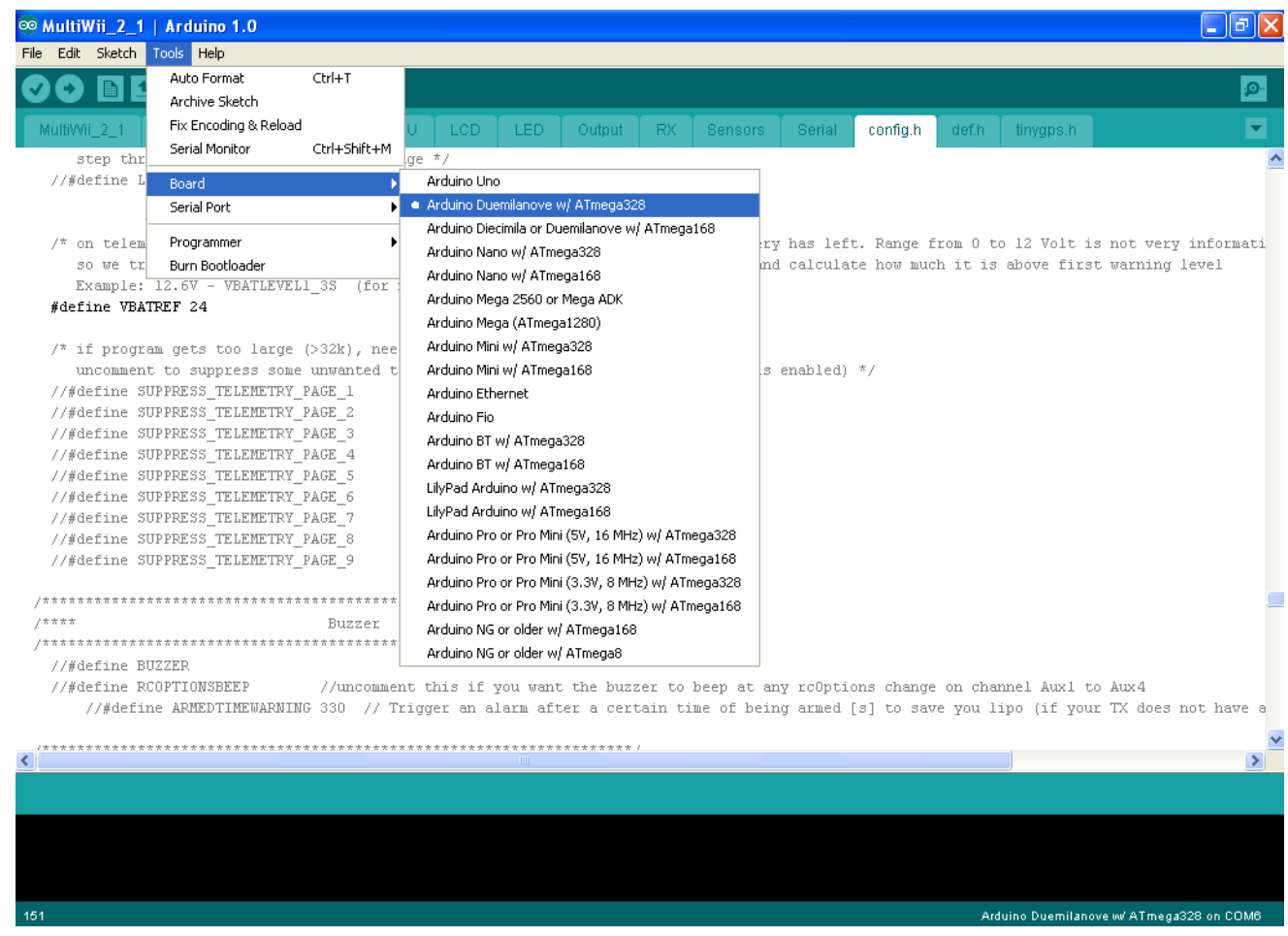

Figure 7.1 Arduino environment

Tools> Programmer- select AVR ISP.

Tools>COM- select the com port in which FTDI programmer is inserted. 


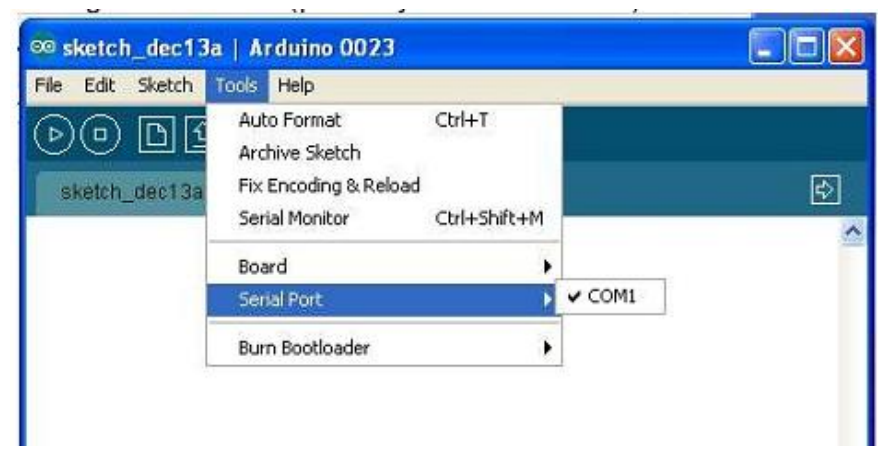

Figure 7.2 Com port selections

After selecting the above settings we can hit the 'Upload' button on the Arduino platform.

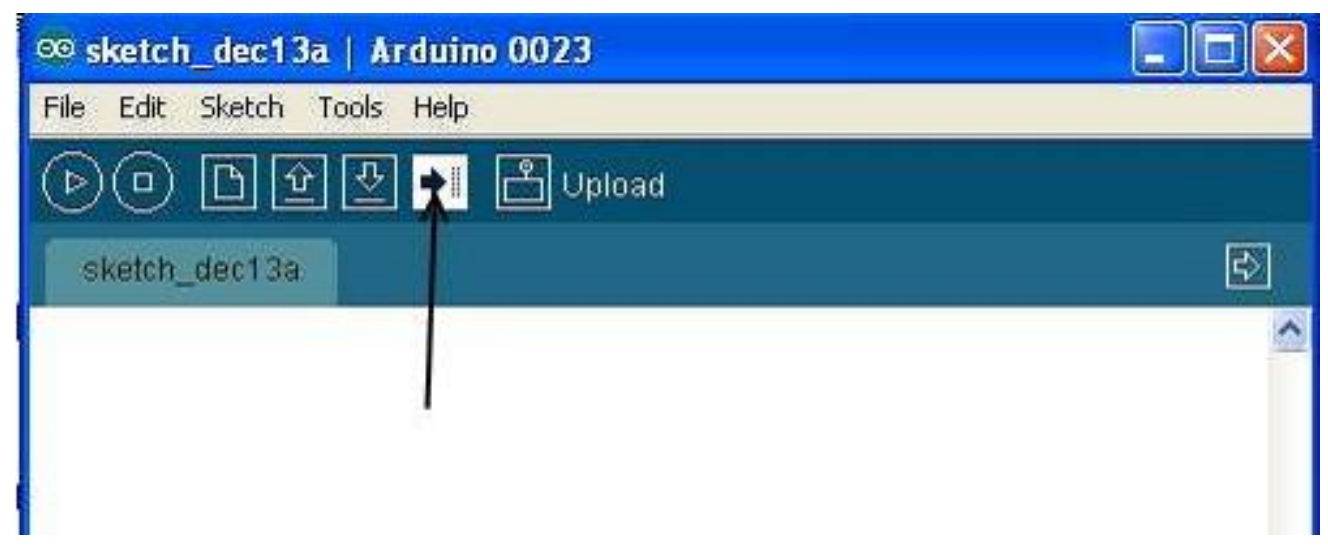

Figure 7.3 Upload click

When we click upload button compilation of program takes place and also uploading of program also takes place which is shown by a progress bar at the bottom of screen.

\subsection{Configuration of Quadcopter}

We can configure the Quadcopter to change the settings in Multiwiiconf_2_1 file which we get when we download Multiwii code. The configuration screen looks as 


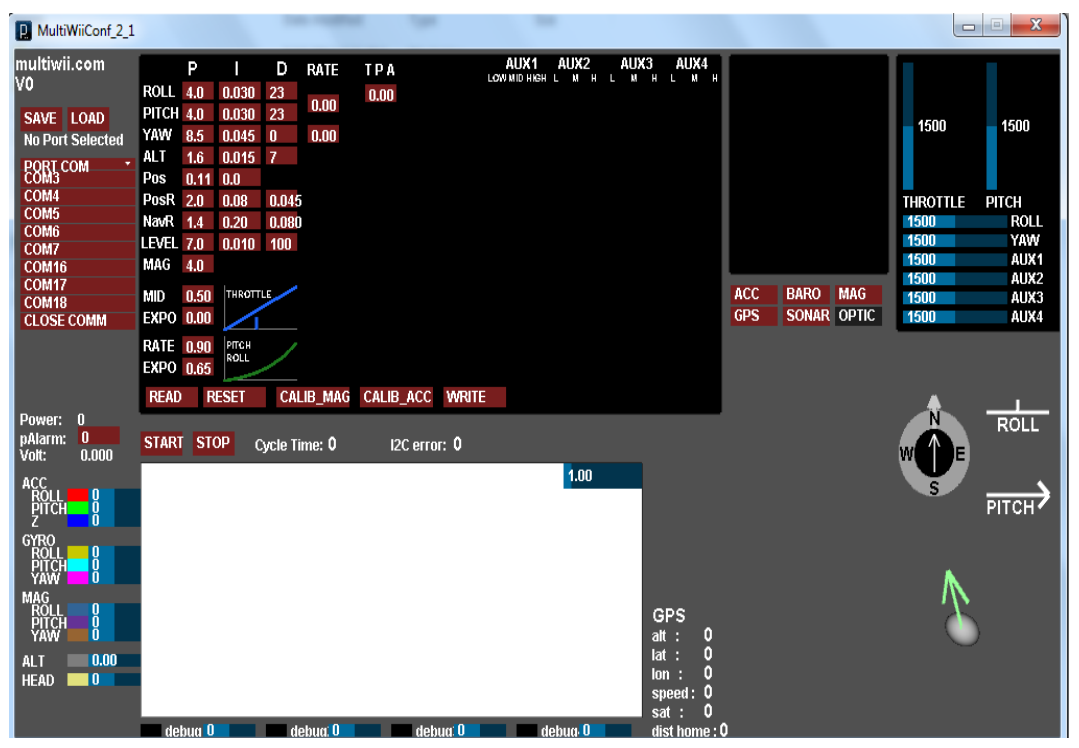

Figure 7.4 Multiwiiconfig screen

- In this first we need to enable the COM port in which the FTDI is inserted.

- Click on 'start' button to read the status of Quadcopter.

- We can virtually have a look of our Quadcopter in configuration screen.

- We can click 'read' to read the PID values of Quadcopter.

\subsubsection{Calibrating accelerometer}

- Keep the Quadcopter and flight control board on a level surface.

- Click 'CALIB_ACC' to calibrate accelerometer.

- After this the ACC Roll and Pitch will show ' 0 ' value and $\mathrm{Z}$ axis has some value of around '250' which indicates the that Quadcopter is at some altitude.

\subsubsection{Calibrating magnetometer}

- Click 'CALIB_MAG' to calibrate magnetometer.

- As soon as we click the button the led on board the LED blinks rapidly -you have 30 secs to rotate the board $360^{\circ}$ degrees in all axes $\mathrm{X}, \mathrm{Y}$ and $\mathrm{Z}$ - when the LED stops blinking it will save all the MAG tilt errors to EPROM.

\subsubsection{Understanding PID}

Once downloaded in the Arduino board, the software set the default settings during the initial start. These settings are appropriate for a configuration similar to 
mine (motors / ESCs / propellers / weight).However, another configuration will probably require other parameters to be optimal.

For instance, if you want to use a larger multicopter for FPV. The multicopter uses a closed controlled loop to ensure its stability and maneuverability.

Like most multirotor, it is a Proportional-Integral-Derivative (PID) regulator. This controller is translated into software code lines in the Arduino and tries to correct the error calculated between a measurement at the controller output (measured by the gyros) and an input set point (position of the stick), using appropriate action to adjust the output of the process (command to motors).

PID controller involves three separate parameters: the term Proportional, the term Integral and the term Derivative. The variation of each of these parameters alters the effectiveness of the stabilization.

Applied to a multirotor, the coefficients of these parameters can be translated by their behavior:

- Proportional coefficient: alone, it may achieve stabilization. This coefficient determines the importance of action on the engines in relation with the values measured by the gyroscopes. The higher the coefficient, the higher the Quadcopter seems more "rigid" versus angular deviation. If it is too low, the multicopter will appear soft and will be harder to keep steady. One can "feel" this setting by handling the Quadcopter and trying to change its orientation: the higher the parameter, the higher the opposition is important. In practice, this parameter must be set alone and increased up to be the limit for obtaining small oscillations. If too high, the system becomes unstable by amplifying the oscillations.

- Integral coefficient: this coefficient can increase the precision of the angular position. In practice, when the Quadcopter is disturbed and its angle changes, the term Integral remembers the disruption and applies a correction to the engines to get the right angle. We can see this term as a heading hold factor. Typically if you take handfuls the multicopter and try to force it into a position, the engines will continue for some time to counteract the action. Without this term, the opposition 
does not last as long. This way, the angular position can be steady and accurate even with irregular wind, or during ground effect. However, the increase of this ratio often involves a reaction speed decrease and a decrease of the Proportional coefficient as a consequence. Compared to the conventional PID algorithm, I decided to cancel this term in the presence of strong angular variations. This strategy allows a safer behavior in case of looping or hard shaking.

- Derivative coefficient: this coefficient allows the Quadcopter to reach more quickly the requested attitude. In practice it will amplify the reaction speed of the system, and in certain cases and increase of the Proportional term. By cons, this parameter induces more noise.

By default, at the first startup, the Quadcopter is initialized with coefficient values that should be quite ok.

The multicopter uses 3 PID loops with their own P I D coefficients, one per axis.

\subsubsection{Tuning PID settings}

- Click 'Read' to read the PID values of Roll, Pitch, Yaw.

- Connect the battery to the Quadcopter.

- Arm (Oning) the Quadcopter using digital switch on Tx.

- Hold the Quadcopter in one of your hands and be careful while holding it.

- Increase the throttle on Tx so that the Quadcopter is not in a position to hover and no weight of Quadcopter is on the person holding it.

- Tilt the Quadcopter giving it some pitch angle and observe whether it is acting against to motion of your hand if not change the PID values accordingly with the properties of PID.

- Continue the above step for Roll and Yaw angles too.

- At last we can obtain optimum PID settings for the Quadcopter. 


\section{CHAPTER 8}

\section{FPV}

\subsection{Introduction}

First-person view (FPV), also known as remote-person view (RPV), or simply video piloting, is a method used to control a radio controlled model vehicle from the driver or pilot's view point. Most commonly it is used to pilot an unmanned aerial vehicle (UAV) or a radio-controlled aircraft. The vehicle is either driven or piloted remotely from a first person perspective via an onboard camera, fed wirelessly to virtual reality goggles or a video monitor.

First-person view (FPV) flight is a type of remote-control (RC) flying that has grown in popularity in recent years. It involves mounting a small video camera and analog television transmitter on an RC aircraft and flying by means of a live video down-link, commonly displayed on video goggles or a portable LCD screen. When flying FPV, the pilot sees from the aircraft's perspective, and does not even have to look at the model. As a result, FPV aircraft can be flown well beyond visual range, limited only by the range of the remote control and video transmitter. FPV became increasingly common throughout the 2000s and early 2010s. It is currently one of the fastest growing activities involving $\mathrm{RC}$ aircraft, and has given rise to a small but growing industry providing products specifically designed for FPV use. 


\subsection{Equipment}

There are two primary components of an FPV setup - the airborne component and the ground component (typically called a ground station). A basic FPV system consists of a camera and video transmitter on the aircraft and a video receiver and a display on the ground. More advanced setups commonly add in specialized hardware, including on-screen displays with GPS navigation and flight data, stabilization systems, and autopilot devices with "return to home" capability-allowing the aircraft to fly back to its starting point on its own in the event of a signal loss. On-board cameras can be equipped with a pan and tilt mount, which when coupled with video goggles and "head tracking" devices creates a truly immersive, first-person experience, as if the pilot was actually sitting in the cockpit of the RC aircraft.

Ground stations can be equipped with high gain antennas and automatic antenna tracking systems to provide for maximum range on the video link.

\subsection{Radio frequencies}

Video transmitters typically operate at a power level between $200 \mathrm{~mW}$ and $1500 \mathrm{~mW}$. The most common frequencies used for video transmission are $900 \mathrm{MHz}$, 1.2 $\mathrm{GHz}, 2.4 \mathrm{GHz}$, and $5.8 \mathrm{GHz}$.

We have equipped our Quadcopter with a CCD camera as specified in specifications in chapter 4 . To the camera we have connected $0.9 \mathrm{GHz}$ four channels Tx to transmit the video.

At the ground station we have a 12 channel $\mathrm{Rx}$ with antenna to receive the video signal and from the Rx we have a RCA cable and that RCA cable can be connected to Television or any display device to view the video.

\subsection{Ready to fly}

After equipped with all components as specified in chapter 4 and configuring it as in chapters 6,7 we are ready to fly the quadcopter! 


\section{CHAPTER 9}

\section{ACHIEVEMENTS}

\subsection{Achievements}

- After flying our Quadcopter we achieved some goals of our task

- Our quad can hover and maneuver for a time of 6minutes with the battery (chapter 4) we used.

- Our quad can stably fly and we have achieved height of over 30feet (we can also get more height but we did not go beyond this due to some air restrictions).

- We have also got the first person view from the camera we equipped on quad over the distance of $200 \mathrm{~m}$.

\subsection{Pictures of flight}

We had taken some snapshots of our flight.

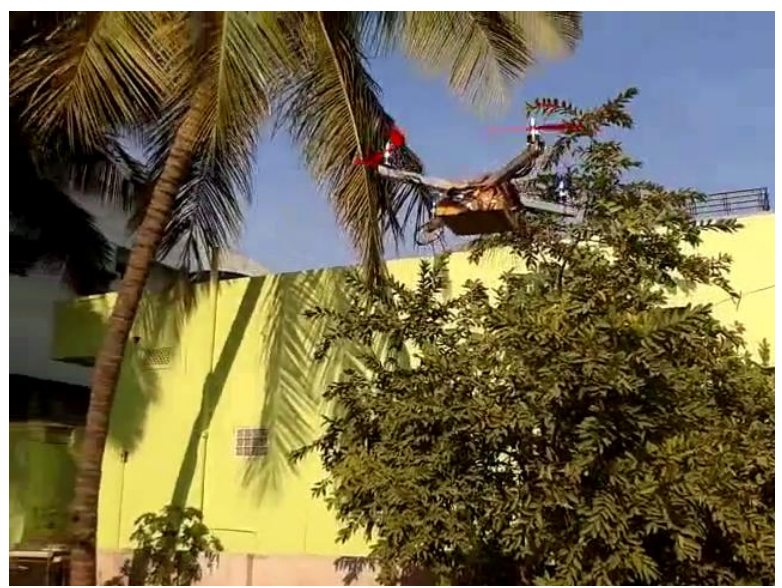

Figure 9.1

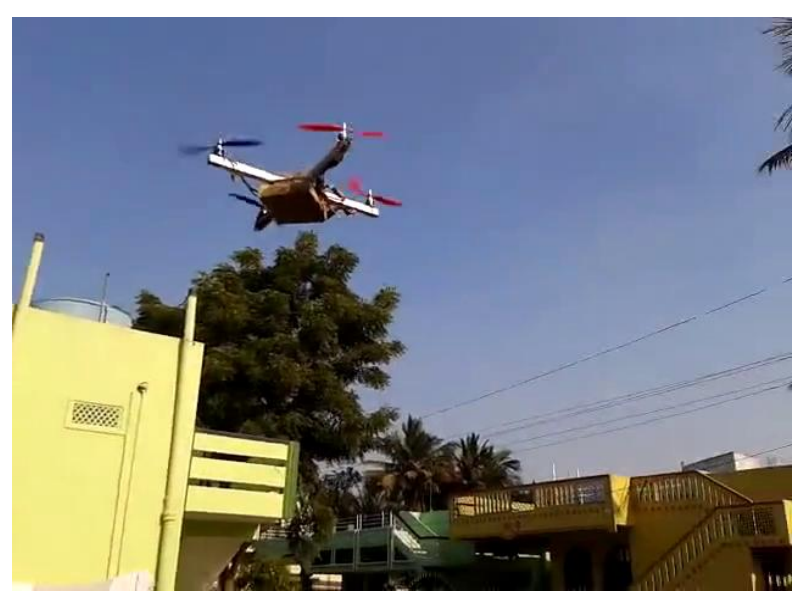

Figure 9.2 

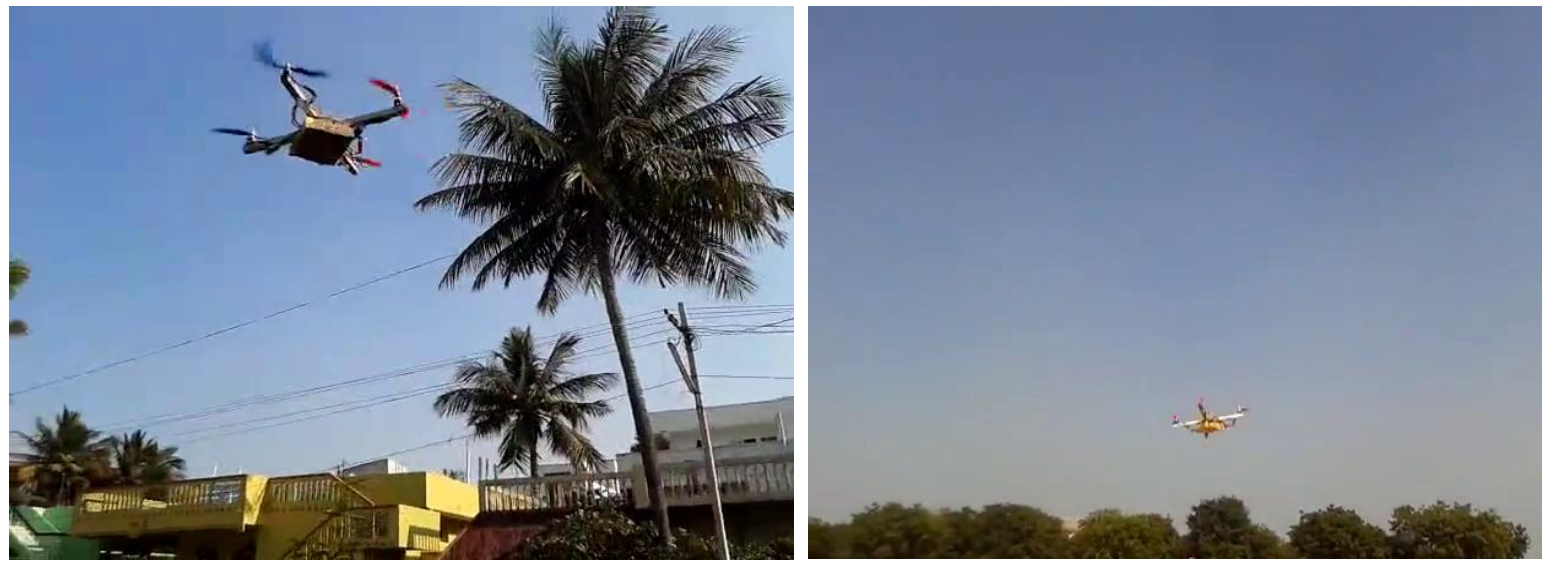

Figure 9.3 Figure 9.4
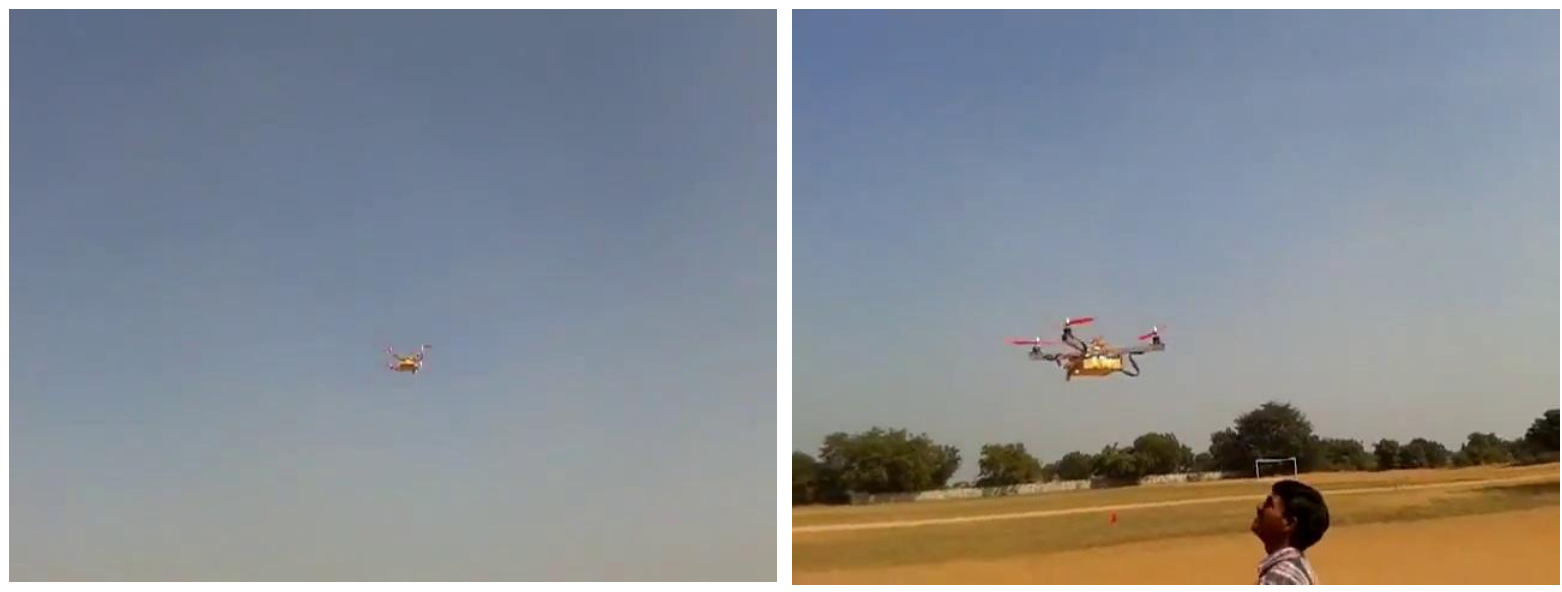

Figure 9.5 Figure 9.6

Some snapshots of our video from camera we mounted on quadcopter.

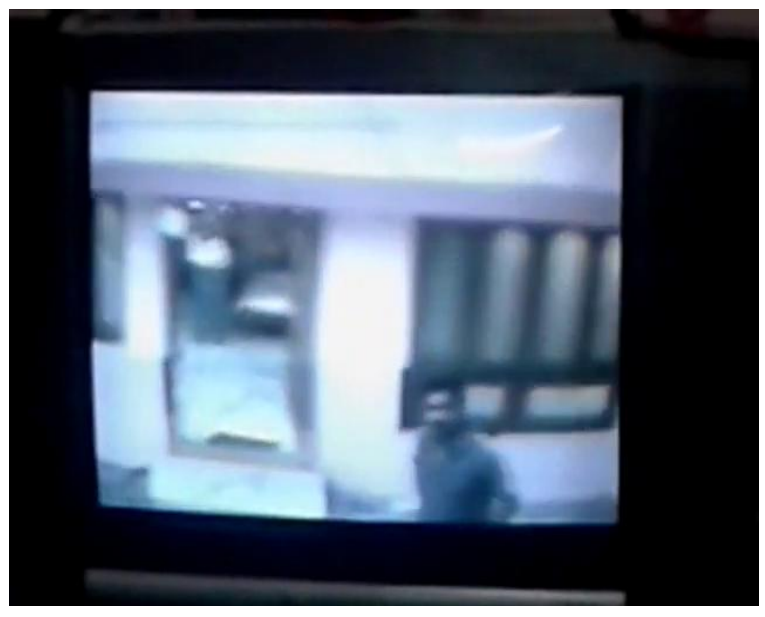

Figure 9.7

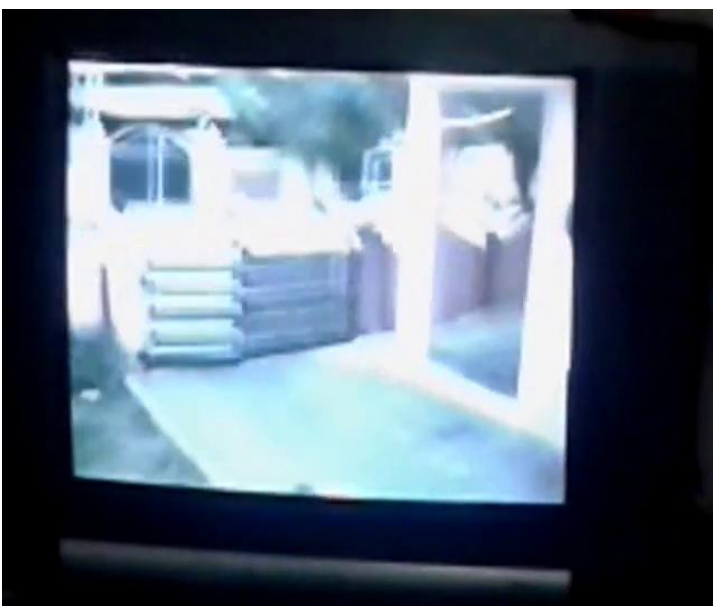

Figure 9.8 


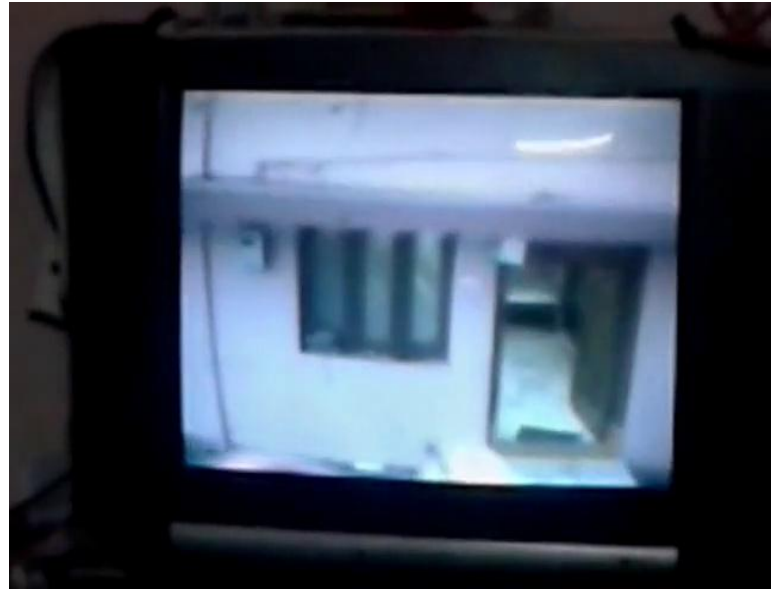

Figure 9.9

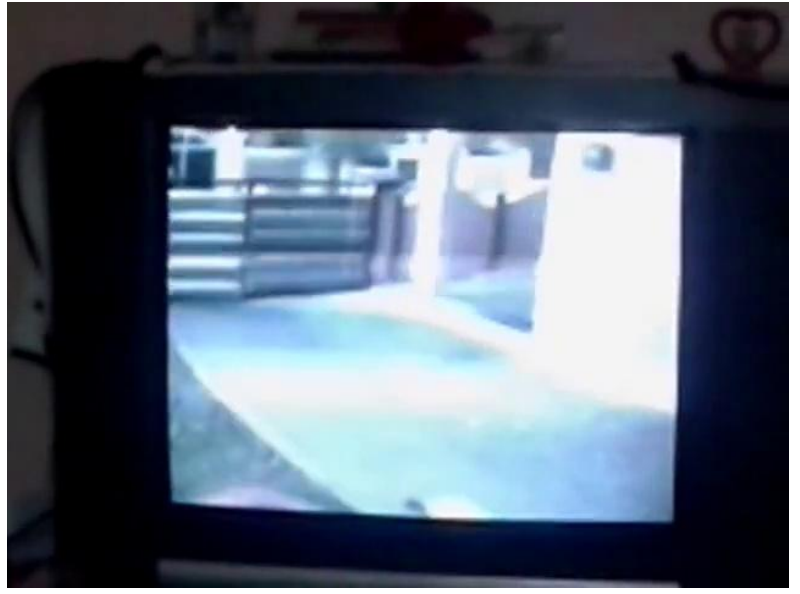

Figure 9.10

\subsection{Videos of flight}

You can view videos of our flight on YouTube

www.youtube.com (channel: Gopi Krishna) 
'Experience is simply the name we give our mistakes.'

-Oscar Wilde

\section{CHAPTER 10}

\section{FURTHER DEVELOPMENT AND ADVANCEMENTS}

\subsection{Further developments}

Albeit we worked hard to develop our quadcopter without flaws, we found some problems during our flight.

So we present some further developments to our vehicle for smooth running and for perfect execution of task.

We have used aluminium frame to build our vehicle. Although it is very less in weight it is not damping the vibrations caused by the motors and these vibrations are tending their effect on the sensors (accelerometers, gyros) we used on the flight control board.

So due to the effect of vibrations on sensors, the sensors are giving wrong orientation (may be very minute, but in aerial vehicles it is significant) to the microcontroller and indeed there is some fluctuations in stability of vehicle which we did not want to prevail.

So to decrease the effect of vibrations we should use 'carbon fibre' instead of aluminium to damp the maximum vibrations. 
In extension with carbon fiber frame we should take necessary steps to damp the vibrations of motors by using some rubber like material down the motor and mount the motor tightly and perfectly.

\subsection{Advancements}

With the motors we have used, our quadcopter cannot make a 180degree turn and hover, so it make it work like that we have to use motors and propellers in such a way that when it turn 180 degrees the propellers should also change their pitch so we have to have a mechanism at the motor to make this.

Our quadcopter can handle the natural disasters like earthquakes, liminic eruptions, heat waves and other disasters may be natural or manmade.

In order to sustain hydro disasters we have to make some necessary advancement to the quadcopter by providing some water resistant material to protect the electrical connections, we can use materials like polyester fleece, plastic etc.

As we go for miniature vehicles for agility we are losing the pay load capacity of vehicle. So in order to increase the payload capacity of vehicles we can go for 'swarm'.

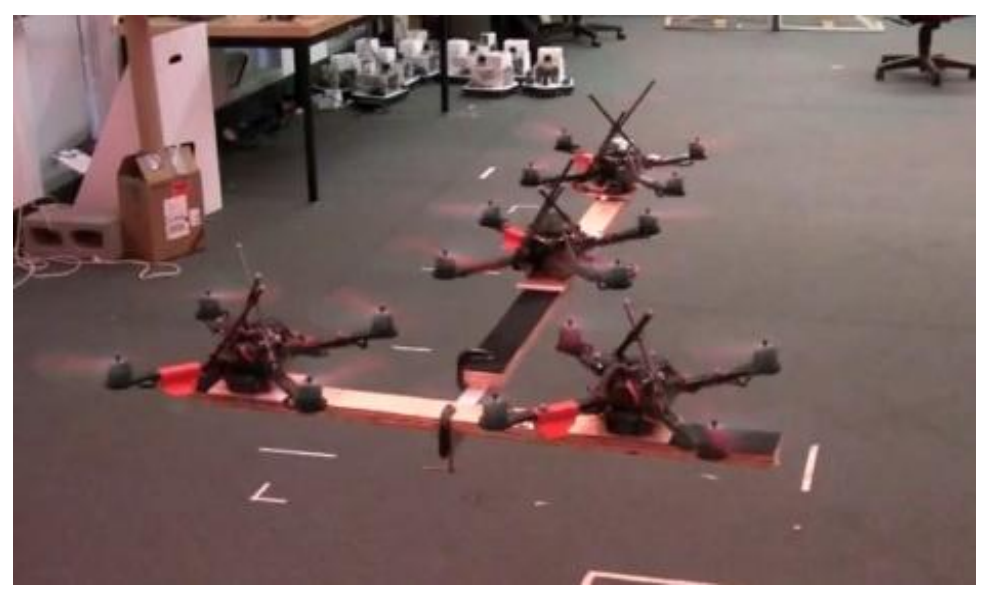

Figure 10.1 To increase payload

When we go for group of quadcopters we should think of communication inbetween them. When we look to nature, we see ants carrying food to their nest, so when they are carrying their food there is no explicit communication between ants and 
there is no centralized communication too. But they communicate implicitly in a decentralized way.

So, learning from nature we have to make the swarm of quads to communicate with each other and develop a formation to carry the payload required.

We have different algorithms for these swarm vehicles as proposed by Vijay Kumar and his team in his paper 'Towards A Swarm of Agile Micro Quadrotors'.

The algorithms include:

- Formation flight:

Flying in formation reduces the complexity of generating trajectories for a large team of vehicles to generating a trajectory for a single entity. If the controllers are well-designed, there is no need to explicitly incorporate collision avoidance between vehicles.

- Time-Separated Trajectory Following

Another way to reduce the complexity of the trajectory generation problem is to require all vehicles to follow the same team trajectory but be separated by some time increment.

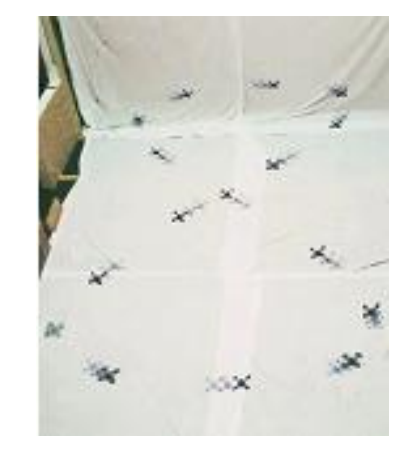

Figure 10.28 formation

Some images for swarm of quadcopters: 


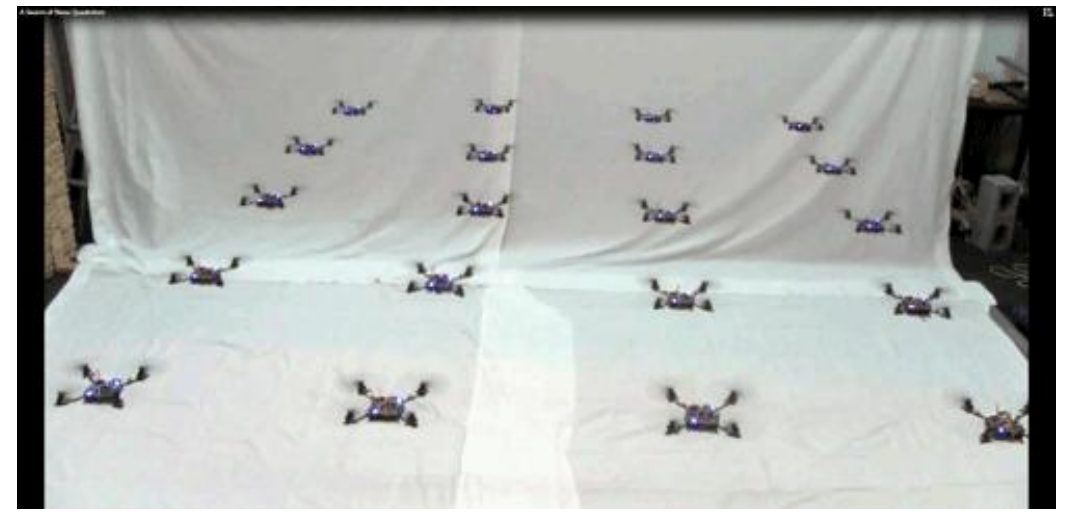

10.3 Swarm of quadcopters

Not only in disasters, we can also make use of these type of unmanned vehicles to reach out into the region where man cannot reach.

In the above aspect we can send these type of vehicles with some sophisticated sensors to planet like 'Mars' for exploration of Red planet.

Not only we can think of controlled quadcopter but also we can think of autonomous vehicles. For indoor flights we can make quadcopter autonomous by placing some excellent motion capturing systems around it to track the exact position of vehicle.

For outdoor autonomous flights we can make use of GPS, Microsoft Kinect or other laser systems for obstacle detection. 


\section{APPENDIX}

The details of cost of components we used (from www.hobbyking.com)

\begin{tabular}{|c|c|c|c|c|}
\hline Code & Qty & Description & Unit price & Total \\
\hline NTM2826-1000 & 4 & NTM Prop Drive 28-26 1000KV / 315W & $\$ 15.99$ ea & $\$ 63.96$ \\
\hline NTM28-ACC & 4 & NTM Prop Drive 28 Series Accessory Pack & $\$ 1.89$ ea & $\$ 7.56$ \\
\hline 9171000077 & 4 & NTM 28 Motor Mount Spacer/Stand Off $2 \mathrm{~mm}$ (4pc) & $\$ 0.73$ ea & $\$ 2.92$ \\
\hline $\mathrm{HKz} 30 \mathrm{~A}$ & 4 & HobbyKing 30A BlueSeries Brushless Speed Controller & $\$ 10.47$ ea & $\$ 41.88$ \\
\hline T2200.3S.20 & 2 & Turnigy 2200 m Ah 3S 20C Lipo Pack & $\$ 7.89 \mathrm{ea}$ & $\$ 15.78$ \\
\hline TGV-Detector & 1 & TURNIGY $3 \sim 8 S$ Voltage Detector & $\$ 2.49$ ea & $\$ 2.49$ \\
\hline $601 \mathrm{~A}-2 \mathrm{PParal}$ & 1 & XT60 Harness for 2 Packs in Parallel (1pc) & $\$ 1.97$ ea & $\$ 1.97$ \\
\hline Turnigy-3S & 1 & Turnigy balancer \& Charger 2S-3S & $\$ 4.49$ ea & $\$ 4.49$ \\
\hline 017000059 & 1 & $10 \times 4.5$ SF Props $2 \mathrm{pc}$ Standard Rotation/2 pc RH Rotation (Blue) & $\$ 3.14 \mathrm{ea}$ & $\$ 3.14$ \\
\hline 017000061 & 1 & 10x4.5 SF Props 2pc Standard Rotation/2 pc RH Rotation (Red) & $\$ 3.14 \mathrm{ea}$ & $\$ 3.14$ \\
\hline AM $1001 \mathrm{~A}$ & 2 & PolyMax $3.5 \mathrm{~mm}$ Gold Connectors 10 PAIRS (20PC) & $\$ 1.59$ ea & $\$ 3.18$ \\
\hline XT60 & 1 & Nylon XT60 Connectors Male/Female ( 5 pairs) GENUINE & $\$ 3.19$ ea & $\$ 3.19$ \\
\hline OR009-00506 & 1 & M3 x $20 \mathrm{~mm}$ Nylon Screws (10pcs/bag) & $\$ 1.07 e a$ & $\$ 1.07$ \\
\hline OR009-01502 & 1 & M3 Nylon Nut (10pcs/bag) & $\$ 0.69$ ea & $\$ 0.69$ \\
\hline HK-6DF-M2 & 1 & HobbyKing HK6S 2.4 Ghz FHSS 6 Ch Tx \& Rx (Mode 2) & $\$ 28.41 \mathrm{ea}$ & $\$ 28.41$ \\
\hline OR013A-02301 & 1 & Non-Adhesive Velcro (1 Set) & $\$ 1.44 \mathrm{ea}$ & $\$ 1.44$ \\
\hline FS-L001 & 1 & Hobby King $2.4 \mathrm{Ghz} 6 \mathrm{Ch}$ Tx USB Cable & $\$ 2.99$ ea & $\$ 2.99$ \\
\hline OR011-02001 & 1 & Reinforced Plastic Undercarriage (Pair) STRONG & $\$ 0.99$ ea & $\$ 0.99$ \\
\hline 9171000009 & 1 & $900 \mathrm{MHZ} 200 \mathrm{~mW}$ Tx/Rx \& 1/3-inch CCD Camera NTSC 520TVL & $\$ 69.47 \mathrm{ea}$ & $\$ 69.47$ \\
\hline Q005 & 1 & FPV Fiberglass Pan-Tilt Camera Mount L-Size & $\$ 4.99 e a$ & $\$ 4.99$ \\
\hline HXT900 & 1 & HXT900 9g / $1.6 \mathrm{~kg} / .12 \mathrm{sec}$ Micro Servo & $\$ 2.69 \mathrm{ea}$ & $\$ 2.69$ \\
\hline
\end{tabular}

HS Code: 9503099

MADE IN CHINA

HOBBY PARTS

INVOICE TOTAL: \$266.44USD

SHIPPING: \$77.53USD

(From www.goodluckbuy.com)

\begin{tabular}{|c|c|c|c|}
\hline Description & Price & Quantity & Amount \\
\hline $\begin{array}{l}\text { FTDI Basic Breakout Arduino USB-TTL } 6 \text { PIN } 3.3 \\
5 \text { V for MWC MultiWii Lite /SE } \\
77995\end{array}$ & $\$ 6.56$ & 1 & $\$ 6.56$ \\
\hline \multirow[t]{5}{*}{$\begin{array}{l}\text { MWC MultiWii SE Standard Edition 4-axis X-mode } \\
\text { Flight Control Board QUADX } \\
77993\end{array}$} & $\$ 46.72$ & 1 & $\$ 46.72$ \\
\hline & & Item total & $\$ 53.28$ \\
\hline & & Tax & $\$ 0.00$ \\
\hline & & Packaging & $\$ 1.60$ \\
\hline & & Total & $\$ 54.88$ USD \\
\hline
\end{tabular}

Total: \$ 398.85 USD 


\section{References}

'Towards A Swarm of Agile Micro Quadrotors' by Alex Kushleyev, Daniel Mellinger, Vijay Kumar, GRASP Lab, University of Pennsylvania

Datasheets: ATMEGA328P, ITG3205, BMA180, HMC5883L

www.multiwii.com - the site for open source code on multirotors

www.wikipedia.com - for enormous data related to concepts of aerodynamic lift

www.hobbyking.com - the site to buy different Radio Controlled (RC) components

www.goodluckbuy.com - the site to buy flight control board

$\underline{\text { www.arduino.cc }}$ - the site for Arduino software

$\underline{\text { www.ecalc.ch }}$ - the site for calculations of multirotors

www.rcgroups.com- the forum for various discussions on multirotors

www.blog.tkjelectronics.dk - the blog to know basics of multirotors

www.ted.com- for video of Vijaykumar on autonomous quadrotors 